\title{
Dynamics of transcendental Hénon maps
}

\author{
Leandro Arosio' ${ }^{1}$. Anna Miriam Benini ${ }^{1}$. \\ John Erik Fornæss ${ }^{2}$. Han Peters ${ }^{3}$
}

Received: 25 May 2017 / Revised: 6 December 2017 / Published online: 13 January 2018 (C) The Author(s) 2018. This article is an open access publication

\begin{abstract}
The dynamics of transcendental functions in the complex plane has received a significant amount of attention. In particular much is known about the description of Fatou components. Besides the types of periodic Fatou components that can occur for polynomials, there also exist so-called Baker domains, periodic components where all orbits converge to infinity, as well as wandering domains. In trying to find analogues of these one dimensional results, it is not clear which higher dimensional transcendental
\end{abstract}

Communicated by Ngaiming Mok.

Leandro Arosio and Anna Miriam Benini were supported by the SIR Grant "NEWHOLITE-New methods in holomorphic iteration" No. RBSI14CFME. Han Peters was supported by the NFR Grant No. 10445200 .

Part of this work was done during the international research program "Several Complex Variables and Complex Dynamics" at the Center for Advanced Study at the Academy of Science and Letters in Oslo during the academic year 2016/2017.

$\bowtie$ Han Peters

hanpeters77@gmail.com

Leandro Arosio

arosio@mat.uniroma2.it

Anna Miriam Benini

ambenini@gmail.com

John Erik Fornæss

john.fornass@ntnu.no

1 Dipartimento Di Matematica, Università di Roma "Tor Vergata", Rome, Italy

2 Department of Mathematical Sciences, NTNU, Trondheim, Norway

3 Korteweg de Vries Institute for Mathematics, University of Amsterdam, Amsterdam, The Netherlands 
maps to consider. In this paper we find inspiration from the extensive work on the dynamics of complex Hénon maps. We introduce the family of transcendental Hénon maps, and study their dynamics, emphasizing the description of Fatou components. We prove that the classification of the recurrent invariant Fatou components is similar to that of polynomial Hénon maps, and we give examples of Baker domains and wandering domains.

Mathematics Subject Classification $32 \mathrm{H} 50 \cdot 37 \mathrm{~F} 50 \cdot 37 \mathrm{~F} 10$

\section{Contents}

1 Introduction . . . . . . . . . . . . . . . . . . . . . . . 854

2 The definition of the Fatou set . . . . . . . . . . . . . . . . . . . . . . . . 857

3 Invariant subsets . . . . . . . . . . . . . . . . . . . . . . . . . . 860

3.1 Periodic points . . . . . . . . . . . . . . . . . . . . 860

3.2 Invariant algebraic curves . . . . . . . . . . . . . . . . . . . . 862

4 Classification of recurrent components . . . . . . . . . . . . . . . . . . . 864

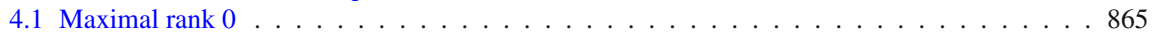

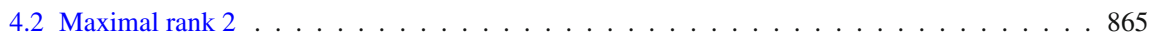

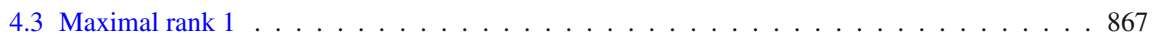

4.4 Transcendental Hénon maps . . . . . . . . . . . . . . . . . . . . . . . . 874

5 Baker domain . . . . . . . . . . . . . . . . . . . . . . . . . . . 874

6 Escaping wandering domain . . . . . . . . . . . . . . . . . . . . . . 881

7 Oscillating wandering domain . . . . . . . . . . . . . . . . . . . 886

7.1 Finding the new oscillation . . . . . . . . . . . . . . . . . . . . 888

7.2 Connecting the orbits via the contracting detour . . . . . . . . . . . . . . . . . . 889

7.3 Complex structure . . . . . . . . . . . . . . . . . . . . . . . 891

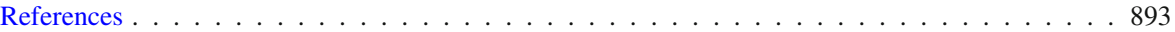

\section{Introduction}

Our goal is to combine ideas from two separate areas of holomorphic dynamics: the study of transcendental dynamics on the complex plane, and the study of polynomial Hénon maps in $\mathbb{C}^{2}$. Recall that a polynomial Hénon map is a map of the form

$$
F:(z, w) \mapsto(f(z)-\delta w, z),
$$

where $f$ is a polynomial of degree at least 2, and $\delta$ is a non-zero constant. Here we consider maps of the same form, but where $f$ is a transcendental entire function. We call such $F$ a transcendental Hénon map, and it is easy to see that $F$ is a holomorphic automorphism of $\mathbb{C}^{2}$ with constant Jacobian determinant $\delta$. Special cases of transcendental Hénon maps, namely transcendental perturbations of polynomial Hénon maps, have been first considered in [13].

The main reason for considering transcendental Hénon maps and not arbitrary entire maps in $\mathbb{C}^{2}$ is that the space of entire maps is too large. Even the class of polynomials maps in two complex variables is often considered too diverse to study the dynamics of these maps all at the same time. On the other side, the family of polynomial automorphisms of $\mathbb{C}^{2}$ has received a large amount of attention. It portrays a wide 
variety of dynamical behavior, yet it turns out that this class of maps is homogeneous enough to describe its dynamical behavior in detail. A result of Friedland and Milnor [22] implies that any polynomial automorphism with non-trivial dynamical behavior is conjugate to a finite composition of polynomial Hénon maps. It turns out that finite compositions of polynomial Hénon maps behave in many regards similarly to single Hénon maps, and the family of Hénon maps is sufficiently rigid to allow a thorough study of its dynamical behavior.

Very little is known about the dynamics of holomorphic automorphisms of $\mathbb{C}^{2}$, although there have been results showing holomorphic automorphisms of $\mathbb{C}^{2}$ with interesting dynamical behavior, such as the construction of oscillating wandering domains by Sibony and the third named author [20], and a result of Vivas, Wold and the last author [28] showing that a generic volume preserving automorphisms of $\mathbb{C}^{2}$ has a hyperbolic fixed point with a stable manifold which is dense in $\mathbb{C}^{2}$. Transcendental Hénon maps seems to be a natural class of holomorphic automorphisms of $\mathbb{C}^{2}$ with non-trivial dynamics, restrictive enough to allow for a clear description of its dynamics, but large enough to display interesting dynamical behaviour which does not appear in the polynomial Hénon case.

We classify in Sect. 4 the invariant recurrent components of the Fatou set of a transcendental Hénon map, that is, components which admits an orbit accumulating to an interior point. Invariant recurrent components have been described for polynomial Hénon maps in [5]; our classification holds not only for transcendental Hénon maps but also for the larger class of holomorphic automorphisms with constant Jacobian. Moreover, using the fact that $f$ is a transcendental holomorphic function, we obtain in Sect. 3 results about periodic points and invariant algebraic curves. We show that the set $\operatorname{Fix}\left(F^{2}\right)$ is discrete, and (if $\delta \neq-1$ ) that $F$ admits infinitely many saddle points of period 1 or 2 , which implies that the Julia set is not empty. We also show that there is no irreducible invariant algebraic curve (the same was proved by Bedford-Smillie for polynomial Hénon maps in [4]). The dynamical behavior can be restricted even further by considering transcendental Hénon maps whose map $f$ has a given order of growth. For example, if the order of growth is smaller than $\frac{1}{2}$, then $\operatorname{Fix}\left(F^{k}\right)$ is discrete for all $k \geq 1$.

We then give examples of Baker domains, escaping wandering domains, and oscillating wandering domains. Such Fatou components appear in transcendental dynamics in $\mathbb{C}$, and for trivial reasons they cannot occur for polynomials. The existence of the filtration gives a similar obstruction for polynomial Hénon maps, but this filtration is lost when considering transcendental Hénon maps.

For a transcendental function a Baker domain is a periodic Fatou component on which the orbits converge locally uniformly to the point $\infty$, which is an essential singularity [7]. We give an example in Sect. 5 of a transcendental Hénon map with a two-dimensional analogue: a Fatou component on which the orbits converge to a point at the line at infinity $\ell^{\infty}$, which is (in an appropriate sense) an essential singularity. In one complex variable for any Baker domain there exists an absorbing domain, equivalent to a half plane $\mathbb{H}$, on which the dynamics is conjugate to an affine function, and the conjugacy extends as a semi-conjugacy to the entire Baker domain. In our example the domain is equivalent to $\mathbb{H} \times \mathbb{C}$, and the dynamics is conjugate to an affine map. 
The final part of the paper is devoted to wandering domains. Recall that wandering domains are known not to exist for one-dimensional polynomials and rational maps [30], but they do arise for transcendental maps (see for example [7]). In higher dimensions it is known that wandering domains can occur for holomorphic automorphisms of $\mathbb{C}^{2}$ [20] and for polynomial maps [1], but whether polynomial Hénon maps can have wandering domains remains an open question. We will consider two types of wandering Fatou components, each with known analogues in the one-dimensional setting. We construct in Sect. 6 a wandering domain, biholomorphic to $\mathbb{C}^{2}$, which is escaping: all orbits converge to the point $[1: 1: 0]$ at infinity. The construction is again very similar to that in one dimension. However, the proof that the domain and its forward images are actually different Fatou components is not the proof usually given in one dimension. Instead of finding explicit sets separating one component from another, we give an argument that uses exponential expansion near the boundary of each of the domains.

Finally, we construct in Sect. 7 a transcendental Hénon map $F$ with a wandering domain $\Omega$, biholomorphic to $\mathbb{C}^{2}$, which is oscillating, that is it contains points whose orbits have both bounded subsequences and subsequences which converge to infinity. Up to a linear change of variable, the map $F$ is the limit as $k \rightarrow \infty$ of automorphisms of $\mathbb{C}^{2}$ of the form $F_{k}(z, w):=\left(f_{k}(z)+\frac{1}{2} w, \frac{1}{2} z\right)$, all having a hyperbolic fixed point at the origin. The family $\left(F_{k}\right)$ is constructed inductively using Runge approximation in one variable to obtain an entire function $f_{k+1}$ which is sufficiently close to $f_{k}$ on larger and larger disks, in such a way that the orbit of an open set $U_{0} \subset \mathbb{C}^{2}$ approaches the origin coming in along the stable manifold of $F_{k}$ and then goes outwards along the unstable manifold of $F_{k}$, over and over for all $k \in \mathbb{N}$.

Regarding the complex structure of those Fatou components, in both the Baker domain and the oscillating wandering domain case one encounters the same difficulty. Namely, in both cases one finds a suitable invariant domain $A$ of the Fatou component on which it is possible to construct, using the dynamics of $F$, a biholomorphism to a model space $\left(\mathbb{H} \times \mathbb{C}\right.$ and $\mathbb{C}^{2}$ respectively, where $\mathbb{H}$ denotes the right half-plane). One then needs to prove that the domain $A$ is in fact the whole Fatou component, and this is done by using the following plurisubharmonic method: If $A$ is strictly smaller than $\Omega$ then we can construct a plurisubharmonic function $u: \Omega \rightarrow \mathbb{R} \cup\{-\infty\}$ for which the submean value property is violated at points in $\partial A \cap \Omega$. We note that a somewhat similar argument was given by the third author in [18], and we believe that this method can be applied in a variety of similar circumstances.

It is important to point out that for an entire map $F: \mathbb{C}^{2} \rightarrow \mathbb{C}^{2}$ there are two natural definitions of the Fatou set, which correspond to compactifying $\mathbb{C}^{2}$ either with the onepoint compactification $\widehat{\mathbb{C}^{2}}$, or with $\mathbb{P}^{2}$. In one dimension the two Fatou sets coincide, and the same is true for polynomial Hénon maps, since by the existence of the filtration all forward orbits that converge to infinity converge to the same point on the line at infinity $\ell^{\infty}=\mathbb{P}^{2} \backslash \mathbb{C}^{2}$. For a general entire self-map of $\mathbb{C}^{2}$ these two definitions can give two different Fatou sets (see Example 2.6). Notice that, if we compactify with $\widehat{\mathbb{C}^{2}}$, any open subset of $\mathbb{C}^{2}$ on which the sequence of iterates $F^{n}$ diverges uniformly on compact subsets would be in the Fatou set regardless of how the orbits go to infinity. This seems to be too weak a definition in two complex variables. We thus define the 
Fatou set compactifying $\mathbb{C}^{2}$ with $\mathbb{P}^{2}$ (which has the additional advantage of being a complex manifold). Section 2 is devoted to this argument.

\section{The definition of the Fatou set}

Let $n \in \mathbb{N}, n \geq 1$ and let $X$ be a complex manifold. There are (at least) two natural definitions of what it means for a family $\mathcal{F} \subset \operatorname{Hol}\left(X, \mathbb{C}^{n}\right)$ to be normal. We denote by $\widehat{\mathbb{C}^{n}}$ the one-point compactification of $\mathbb{C}^{n}$, and with the symbol $\infty$ we denote both the point at infinity and the constant map $z \mapsto \infty$.

Definition 2.1 A family $\mathcal{F} \subset \operatorname{Hol}\left(X, \mathbb{C}^{n}\right)$ is $\mathbb{P}^{n}$-normal if for every sequence $\left(f_{n}\right)$ $\in \mathcal{F}$ there exists a subsequence $\left(f_{n_{k}}\right)$ converging uniformly on compact subsets to $f$ $\in \operatorname{Hol}\left(X, \mathbb{P}^{n}\right)$. In other words, $\mathcal{F}$ is relatively compact in $\operatorname{Hol}\left(X, \mathbb{P}^{n}\right)$.

A family $\mathcal{F} \subset \operatorname{Hol}\left(X, \mathbb{C}^{n}\right)$ is $\widehat{\mathbb{C}}^{n}$-normal if for every sequence $\left(f_{n}\right) \in \mathcal{F}$ which is not divergent on compact subsets there exists a subsequence $\left(f_{n_{k}}\right)$ converging uniformly on compact subsets to $f \in \operatorname{Hol}\left(X, \mathbb{C}^{n}\right)$. This is equivalent to $\mathcal{F}$ being relatively compact in $\operatorname{Hol}\left(X, \mathbb{C}^{n}\right) \cup \infty \subset C^{0}\left(X, \widehat{\mathbb{C}^{n}}\right)$.

Remark 2.2 When $n=1$ the two definitions are equivalent.

A family $\mathcal{F} \subset \operatorname{Hol}\left(X, \mathbb{C}^{n}\right)$ is $\mathbb{P}^{n}$-normal if and only if it is equicontinuous with respect to the Fubini-Study distance on $\mathbb{P}^{n}$. This follows from the Ascoli-Arzelà theorem and from the fact that $\operatorname{Hol}\left(X, \mathbb{P}^{n}\right)$ is closed in $C^{0}\left(X, \mathbb{P}^{n}\right)$. One may think that, similarly, a family $\mathcal{F} \subset \operatorname{Hol}\left(X, \mathbb{C}^{n}\right)$ is $\widehat{\mathbb{C}^{n}}$-normal if and only if it is equicontinuous with respect to the spherical distance $d_{\widehat{\mathbb{C}}^{n}}$ on $\widehat{\mathbb{C}^{n}}$, but this is not the case, as the following example shows.

Example 2.3 For $n \geq 2$, the family $\operatorname{Hol}\left(\mathbb{D}, \mathbb{C}^{n}\right) \cup \infty$ is not closed in $C^{0}\left(\mathbb{D}, \widehat{\mathbb{C}^{n}}\right)$. As a consequence, for a family $\mathcal{F} \subset \operatorname{Hol}\left(\mathbb{D}, \mathbb{C}^{n}\right)$, being relatively compact in $C^{0}\left(\mathbb{D}, \widehat{\mathbb{C}^{n}}\right)$ is not equivalent to being $\widehat{\mathbb{C}^{n}}$-normal.

Proof Let $n=2$. Let $s_{n} \geq 0$ be an increasing sequence of real numbers converging to $\frac{1}{2}$. Let $\alpha_{n} \leq \frac{\pi}{2}$ be a decreasing sequence converging to 0 . We define the Pac-man

$$
P_{n}:=\overline{\mathbb{D}} \backslash\left\{s_{n}+\rho e^{i \theta}: \rho>0,|\theta|<\alpha_{n}\right\} .
$$

Let $r_{n} \geq 0$ be an increasing sequence converging to $\frac{1}{2}$. Let $\beta_{n}$ be a sequence decreasing to 0 . We define the bait

$$
R_{n}:=\overline{\mathbb{D}} \cap\left\{z \in \mathbb{C}:|\operatorname{Im} z| \leq \beta_{n}, \operatorname{Re} z \geq r_{n}\right\}
$$

Clearly

$$
\bigcap_{n \in \mathbb{N}} R_{n}=\left\{z \in \mathbb{C}:|\operatorname{Im} z|=0, \frac{1}{2} \leq \operatorname{Re} z \leq 1\right\},
$$

which we call the slit $S$. 
Fig. 1 The mouth of Pac-man $P_{n+1}$ is contained in bait $R_{n}$

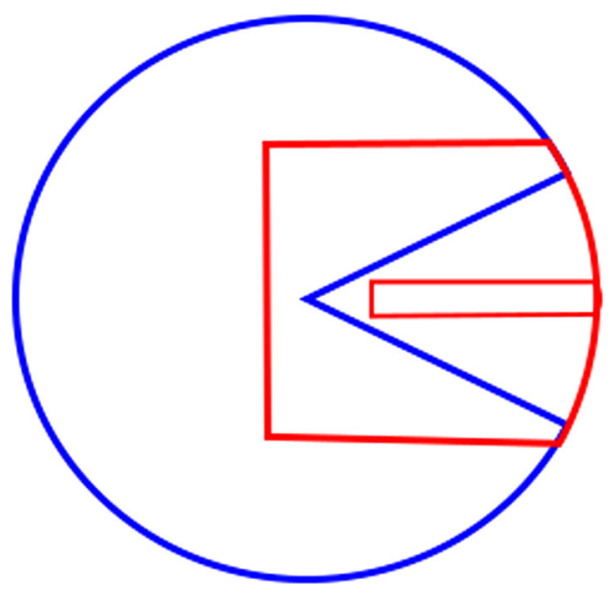

We can choose the sequences $\left(s_{n}\right),\left(\alpha_{n}\right),\left(r_{n}\right),\left(\beta_{n}\right)$ in such a way that

$$
\begin{aligned}
& P_{n} \cap R_{n}=\varnothing \\
& \mathbb{D} \backslash P_{n+1} \subset R_{n} .
\end{aligned}
$$

Notice that this implies that $s_{n} \leq r_{n} \leq s_{n+1}$ for all $n \in \mathbb{N}$. See Fig. 1 for an illustration of a single Pac-man $P_{n+1}$ and two baits $R_{n+1}$ and $R_{n}$.

Let $b_{i}>0$ be a sequence such that for all $n \in \mathbb{N}, x \in \mathbb{C}^{2}$ the following implication holds

$$
\|x\|+\frac{1}{2^{n+1}} \geq \sum_{i=0}^{n-1} b_{i} \Longrightarrow d_{\widehat{\mathbb{C}^{2}}}(x, \infty) \leq \frac{1}{2^{n+1}} .
$$

By using Runge approximation we can define a sequence of holomorphic functions $f_{n}: D(0,1+\epsilon) \rightarrow \mathbb{C}$ such that $\left|f_{n}\right| \leq \frac{1}{2^{n+1}}$ on $P_{2 n}$, and $\operatorname{Re} f_{n} \geq b_{n}$ on $R_{2 n}$, and a sequence of holomorphic functions $g_{n}: D(0,1+\epsilon) \rightarrow \mathbb{C}$ such that $\left|g_{n}\right| \leq \frac{1}{2^{n+1}}$ on $P_{2 n+1}$, and $\operatorname{Re} g_{n} \geq b_{n}$ on $R_{2 n+1}$. For all $n \geq 0$, let $H_{n}: D(0,1+\epsilon) \rightarrow \mathbb{C}^{2}$ be defined as $H_{n}(z)=\sum_{j=0}^{n}\left(f_{n}(z), g_{n}(z)\right)$. Denote

$$
a_{n}:=\max _{z \in \overline{\mathbb{D}}} d_{\widehat{\mathbb{C}^{2}}}\left(H_{n}(z), H_{n-1}(z)\right) .
$$

We claim that $a_{n} \leq \frac{1}{2^{n}}$, and thus the sequence $\left(H_{n}\right)$ converges uniformly on the disk $\overline{\mathbb{D}}$ to a continuous mapping $H: \overline{\mathbb{D}} \rightarrow \widehat{\mathbb{C}^{2}}$ such that $H(\overline{\mathbb{D}} \backslash S) \subset \mathbb{C}^{2}$ and $H(S)=\{\infty\}$. Fix $n \in \mathbb{N}$. If $z \in P_{2 n}$, then $\left|f_{n}(z)\right| \leq \frac{1}{2^{n+1}}$ and $\left|g_{n}(z)\right| \leq \frac{1}{2^{n+1}}$. Thus $\left\|\left(f_{n}(z), g_{n}(z)\right)\right\|$ $\leq \frac{1}{2^{n}}$, which implies that $d_{\widehat{\mathbb{C}^{2}}}\left(H_{n}(z), H_{n-1}(z)\right) \leq \frac{1}{2^{n}}$. If $z \in \mathbb{D} \backslash\left(P_{2 n} \cup R_{2 n}\right)$, then we have that $\operatorname{Re} \sum_{i=0}^{n-1} g_{i}(z) \geq \sum_{i=0}^{n-1} b_{i}$ and $\left|g_{n}(z)\right| \leq \frac{1}{2^{n+1}}$, and thus by (1) both $H_{n}(z)$ and $H_{n-1}(z)$ belong to the ball of radius $\frac{1}{2^{n+1}}$ centered at $\infty$. If $z \in R_{2 n}$, then we have that $\operatorname{Re} \sum_{i=0}^{n-1} f_{i}(z) \geq \sum_{i=0}^{n-1} b_{i}$ and $\operatorname{Re} f_{n}(z) \geq b_{n}$, and thus by (1) both $H_{n}(z)$ and $H_{n-1}(z)$ belong to the ball of radius $\frac{1}{2^{n+1}}$ centered at $\infty$. 
Lemma 2.4 If a family $\mathcal{F} \subset \operatorname{Hol}\left(X, \mathbb{C}^{n}\right)$ is $\mathbb{P}^{n}$-normal, then it is $\widehat{\mathbb{C}}^{n}$-normal.

Proof Let $\left(f_{n}\right)$ be a sequence in $\mathcal{F}$. Since $\mathcal{F}$ is $\mathbb{P}^{n}$-normal there exists a subsequence $\left(f_{n_{k}}\right)$ converging uniformly on compact subsets to a map $f \in \operatorname{Hol}\left(X, \mathbb{P}^{n}\right)$. If there is a point $x \in X$ such that $f(x) \in \ell^{\infty}$, then $f(X) \subset \ell^{\infty}$. Indeed, it suffices to show that $f^{-1}\left(\ell^{\infty}\right)$ is open, and this follows taking an affine chart around $f(y) \in \ell^{\infty}$ in such a way that $\ell^{\infty}=\left\{z_{1}=0\right\}$ and applying Hurwitz theorem to the sequence $\pi_{1} \circ f_{n}$.

Thus, if the sequence $\left(f_{n}\right)$ is not diverging on compact subsets, the subsequence $\left(f_{n_{k}}\right)$ converges uniformly on compact subsets to a map $f \in \operatorname{Hol}\left(X, \mathbb{C}^{n}\right)$.

As a consequence of the previous discussion, for an entire map $F: \mathbb{C}^{n} \rightarrow \mathbb{C}^{n}$ we have two possible definitions of the Fatou set.

Definition 2.5 A point $z \in \mathbb{C}^{n}$ belongs to the $\widehat{\mathbb{C}^{n}}$-Fatou set if the family of iterates $\left(F^{n}\right)$ is $\widehat{\mathbb{C}^{n}}$-normal near $z$. A point $z \in \mathbb{C}^{n}$ belongs to the $\mathbb{P}^{n}$-Fatou set if the family of iterates $\left(F^{n}\right)$ is $\mathbb{P}^{n}$-normal near $z$.

For a polynomial Hénon map, it follows from the existence of the invariant filtration that any forward orbit that converges to infinity must converge to the point $[1: 0$ : $0] \in \ell^{\infty}$. Thus, the two definitions of Fatou set coincide. By Lemma 2.4 the $\mathbb{P}^{n}$-Fatou set is always contained in the $\widehat{\mathbb{C}^{n}}$-Fatou set. If $n>1$ the inclusion may be strict as the following example shows.

Example 2.6 Given an increasing sequence $N_{j} \in \mathbb{N}$, consider the sequence of polynomials

$$
f_{j}(z)=(z-5(j-1))^{N_{j}},
$$

defined respectively on the disks $D_{j}=D(5(j-1), 2)$, where $j \geq 1$. Given a sequence $\epsilon_{j} \searrow 0$, by Runge approximation (see e.g. Lemma 7.4) we can find an entire function $f$ that is $\epsilon_{j}$-close to $f_{j}$ on $\bar{D}_{j}$ for all $j$.

Define the map $F \in \operatorname{Aut}\left(\mathbb{C}^{2}\right)$ by

$$
F(z, w)=(z+5, w+f(z)) .
$$

It follows immediately from the first coordinate that the forward orbit of any point $\left(z_{0}, w_{0}\right)$ converges to infinity, i.e. $\left\|F^{n}\left(z_{0}, w_{0}\right)\right\| \rightarrow \infty$, hence the $\widehat{\mathbb{C}^{2}}$-Fatou set equals all of $\mathbb{C}^{2}$. Moreover, if $\left|z_{0}\right|<1$ then $F^{n}\left(z_{0}, w_{0}\right) \rightarrow[1: 0: 0]$, uniformly on compact subsets. Thus, the domain $\mathbb{D} \times \mathbb{C}$ is contained in a $\mathbb{P}^{2}$-Fatou component.

On the other hand, if the sequence $N_{j}$ increases sufficiently fast, then for $1<\left|z_{0}\right|$ $\leq 2$ we have that $F^{n}\left(z_{0}, w_{0}\right) \rightarrow[0: 1: 0] \in \ell^{\infty}$, again uniformly on compact subsets. It follows that $\mathbb{D} \times \mathbb{C}$ is a $\mathbb{P}^{2}$-Fatou component. Therefore in this example the single $\widehat{\mathbb{C}^{2}}$-Fatou component contains infinitely many distinct $\mathbb{P}^{2}$-Fatou components.

In what follows, we will only consider $\mathbb{P}^{2}$-normality. We will call the $\mathbb{P}^{2}$-Fatou set simply the Fatou set. The Julia set is the complement of the Fatou set. 


\section{Invariant subsets}

\subsection{Periodic points}

If $g$ is a transcendental function or a polynomial Hénon map, then, for each $k \geq 1$, the set Fix $\left(g^{k}\right)$ is discrete. Clearly this statement is not satisfied for holomorphic automorphisms of $\mathbb{C}^{2}$. For example, one can consider any holomorphic conjugate of a rational rotation.

Consider a periodic orbit

$$
\left(z_{0}, w_{0}\right) \mapsto\left(z_{1}, w_{1}\right) \mapsto \cdots \mapsto\left(z_{k}, w_{k}\right)=\left(z_{0}, w_{0}\right)
$$

Since $w_{j+1}=z_{j}$ for each $j$, the first coordinate function of the Hénon map gives the following relations

$$
\left\{\begin{array}{l}
f\left(z_{0}\right)=z_{1}+\delta z_{k-1} \\
f\left(z_{1}\right)=z_{2}+\delta z_{0} \\
\quad \vdots \\
f\left(z_{k-1}\right)=z_{0}+\delta z_{k-2} .
\end{array}\right.
$$

Lemma 3.1 If $F$ is a transcendental Hénon map, then $\operatorname{Fix}(F)$ and $\operatorname{Fix}\left(F^{2}\right)$ are discrete.

Proof The fixed points $(z, w)$ of $F$ satisfy $z=w$ and thus $z=f(z)-\delta z$. Since $f$ is not linear the set of solutions is discrete.

When $k=2$ the system (2) gives

$$
\left\{\begin{array}{l}
f\left(z_{0}\right)=(1+\delta) z_{1} \\
f\left(z_{1}\right)=(1+\delta) z_{0}
\end{array}\right.
$$

When $\delta=-1$ it is immediate that the set of solutions is discrete. When $\delta \neq-1$ the solutions satisfy

$$
\left\{\begin{array}{l}
\frac{f\left(\frac{f\left(z_{0}\right)}{1+\delta}\right)}{1+\delta}=z_{0} \\
z_{1}=\frac{f\left(z_{0}\right)}{1+\delta} .
\end{array}\right.
$$

and again one observes a discrete set of solutions.

Without making further assumptions it is not clear to the authors that $\operatorname{Fix}\left(F^{k}\right)$ is discrete when $k \geq 3$. However, we can show discreteness when we assume that the function $f$ has small order of growth.

Proposition 3.2 Let $F$ be a transcendental Hénon maps such that $f$ has order of growth strictly less than $\frac{1}{2}$. Then $\operatorname{Fix}\left(F^{k}\right)$ is discrete for all $k \geq 1$.

Proof Consider the entire function

$$
g(z):=\frac{f(z)-f(0)}{z} .
$$


Write

$$
m(r):=\inf _{|z|=r}|g(z)|
$$

Since $f$ is assumed to have order of growth strictly less than $\frac{1}{2}$, so does $g$, and Wiman's Theorem [32] implies that there exist radii $r_{n} \rightarrow \infty$ for which $m\left(r_{n}\right) \rightarrow \infty$.

Suppose for the purpose of a contradiction that the solution set in $\mathbb{C}^{k}$ of the system (2) is not discrete. Then there exists an unbounded connected component $V$. Let $n \in \mathbb{N}$ be such that $V$ intersects the polydisk $D\left(0, r_{n}\right)^{k}$. Then $V$ also intersects the boundary $\partial D\left(0, r_{n}\right)^{k}$, say in a point $\left(z_{0}, \ldots, z_{k-1}\right)$. By the symmetry of the equations in (2) we may then well assume that $\left|z_{0}\right|=r_{n}$, and of course that $\left|z_{j}\right| \leq r_{n}$ for $j=1, \ldots, k-1$.

By Wiman's Theorem we may assume that $\left|g\left(z_{0}\right)\right|$ is arbitrarily large, and in particular that $\left|f\left(z_{0}\right)\right|>(1+|\delta|) r_{n}$. But this contradicts the first equation in (2), completing the proof.

We now turn to the question whether transcendental Hénon maps always have periodic points.

Definition 3.3 For a self-map $h$ and for all $n \geq 1$ we denote by $\operatorname{Per}_{n}(h)$ the set of periodic points of $h$ of minimal period $n$.

Recall that if $f$ is an entire transcendental function, then $\operatorname{Per}_{2}(f)$ has infinite cardinality by [6].

Proposition 3.4 If $F(z, w)=(f(z)-\delta w, z)$ is a transcendental Hénon map, then $\operatorname{Fix}(F) \neq \varnothing$ unless $f(z)-z(\delta+1)=e^{h(z)}$ for some holomorphic function $h(z)$. The set $\operatorname{Per}_{2}(F)$ has infinite cardinality if $\delta \neq-1$, and if $\delta=-1$ the set $\operatorname{Per}_{2}(F)$ is not empty if and only if the set $\{f=0\}$ contains at least two points.

Proof Let $Z:=\{f-z(\delta+1)=0\} \subset \mathbb{C}$. Then $\operatorname{Fix}(F)=\left\{(z, z) \in \mathbb{C}^{2}: z \in Z\right\}$.

If $\delta \neq-1$, let $g(z):=\frac{f(z)}{1+\delta}$. By (4), $\operatorname{Per}_{2}(F)$ coincides with

$$
\left\{\left(z_{0}, g\left(z_{0}\right)\right) \in \mathbb{C}^{2}: z_{0} \in \operatorname{Per}_{2}(g)\right\}
$$

which is non-empty and has infinite cardinality. If $\delta=-1$, then

$$
\operatorname{Per}_{2}(F)=\left\{(z, w) \in \mathbb{C}^{2}: z, w \in Z, z \neq w\right\} .
$$

Remark 3.5 Notice that the set $Z$ has finite cardinality if and only if $f(z)-z(\delta+1)=$ $p(z) e^{h(z)}$, where $p$ is a nonzero polynomial $p$ and $h$ is entire function. Thus in all other cases the sets $\operatorname{Fix}(F)$ and $\operatorname{Per}_{2}(F)$ have infinite cardinality.

If $f: \mathbb{C} \rightarrow \mathbb{C}$ is an entire transcendental function, we have additional information on the multiplier of repelling periodic points of period $n \geq 2$. Indeed we have the following theorem [8, Theorem 1.2]: 
Theorem 3.6 Let $g$ be a transcendental entire function and let $n \in \mathbb{N}, n \geq 2$. Then there is a sequence $\left(z_{k}\right)$ in $\operatorname{Per}_{n}(g)$ such that

$$
\left|\left(g^{n}\right)^{\prime}\left(z_{k}\right)\right| \rightarrow \infty \text { as } k \rightarrow \infty
$$

Corollary 3.7 (Non-empty Julia set) If $\delta \neq-1$, then there exist infinitely many saddle points in $\operatorname{Per}_{2}(F)$, and thus the Julia set of $F$ is non-empty.

Proof We have seen that for all $z_{0} \in \operatorname{Per}_{2}(g)$, the point $\left(z_{0}, g\left(z_{0}\right)\right) \in \operatorname{Per}_{2}(F)$. A computation using the explicit form for $F$ gives

$$
d_{\left(z_{0}, g\left(z_{0}\right)\right)} F^{2}=\left(\begin{array}{cc}
f^{\prime}\left(g\left(z_{0}\right)\right) \cdot f^{\prime}\left(z_{0}\right)-\delta & -\delta f^{\prime}\left(g\left(z_{0}\right)\right) \\
f^{\prime}\left(z_{0}\right) & -\delta
\end{array}\right)
$$

Since

$$
\operatorname{det} d_{\left(z_{0}, g\left(z_{0}\right)\right)} F^{2}=\delta^{2}, \quad \operatorname{tr} d_{\left(z_{0}, g\left(z_{0}\right)\right)} F^{2}=f^{\prime}\left(g\left(z_{0}\right)\right) \cdot f^{\prime}\left(z_{0}\right)-2 \delta,
$$

the point $\left(z_{0}, g\left(z_{0}\right)\right)$ is a periodic saddle point for $\left|f^{\prime}\left(g\left(z_{0}\right)\right) \cdot f^{\prime}\left(z_{0}\right)\right|$ sufficiently large (Observe that $g^{\prime}\left(g\left(z_{0}\right)\right) \cdot g^{\prime}\left(z_{0}\right)$, and hence $f^{\prime}\left(g\left(z_{0}\right)\right) \cdot f^{\prime}\left(z_{0}\right)$, can be taken arbitrarily large by Theorem 3.6).

\subsection{Invariant algebraic curves}

It follows from a result of Bedford-Smillie [4] that a polynomial Hénon map does not have any invariant algebraic curve. Indeed, given any algebraic curve, the normalized currents of integration on the push-forwards of this curve converge to the $(1,1)$ current $\mu^{-}$, whose support does not lie on an algebraic curve.

This type of argument is not available for transcendental dynamics. Here we present a different argument.

Theorem 3.8 Let $F$ be a holomorphic automorphism of the form

$$
F:(z, w) \mapsto(f(z)-\delta w, z),
$$

where $f$ is an entire function, and assume that $F$ leaves invariant an irreducible algebraic curve $\{H(z, w)=0\}$. Then $f$ is affine.

As we remarked earlier, the statement is known when $f$ is a polynomial of degree at least 2, so we will assume that $f$ is a transcendental entire function and obtain a contradiction. Let us first rule out the simple case where $\{H=0\}$ is given by a graph $\{z=g(w)\}$ (the following argument actually works for an entire function $g$ ). In that case the invariance under $F$ gives

$$
f(g(w))-\delta w=g \circ g(w) .
$$


Writing $f(z)=g(z)+h(z)$ gives

$$
h \circ g(w)=\delta w
$$

which implies that $g$ and $h$ are invertible and thus affine. But then $f$ is also affine and we are done.

For a graph of the form $\{w=g(z)\}$ we obtain the functional equation

$$
z=g(f(z)-\delta g(z))
$$

which again implies that the function $g$ is affine, and then so is $f$.

For the general case $\{H=0\}$, where we may now assume that we are not dealing with a graph, we will use the following two elementary estimates.

Lemma 3.9 There exist $\left(z_{j}, w_{j}\right) \in\{H=0\}$, with $\left|z_{j}\right| \rightarrow \infty$, for which

$$
\left|f\left(z_{j}\right)\right|>\left|z_{j}\right|^{j}
$$

Proof As we have already shown that $\{H=0\}$ is not a graph, it follows that $\{H=0\}$ intersects all but finitely many lines $\{z=c\}$. The result follows from the assumption that $f$ is transcendental.

We use two forms for the polynomial $H$ :

(1) $H(z, w)=p(w) z^{N_{1}}+\sum_{k=0}^{N_{1}-1} \sum_{\ell=0}^{N_{2}} \alpha_{k, \ell} z^{k} w^{\ell}$.

(2) $H(z, w)=q_{0}(z)+\sum_{\ell=1}^{n} q_{\ell}(z) w^{\ell}$.

Note that $q_{0}$ cannot vanish identically, because otherwise $w$ is a factor of $H$ and the zero set is not irreducible.

Lemma 3.10 There exist d large enough so that if $H(z, w)=0$ for $|z|$ sufficiently large, then $|w|<|z|^{d}$.

Proof If $|w|>|z|^{d}$ for arbitrarily large $|z|$ and $d$, then $|w|^{n}\left|q_{n}(z)\right|$ dominates the other terms in the form (2), so $H(z, w)$ cannot vanish.

Proof of Theorem 3.8 By Lemma 3.9 there exist $\left(z_{j}, w_{j}\right)$ with $z_{j} \rightarrow \infty, H\left(z_{j}, w_{j}\right)=$ 0 and $\left|f\left(z_{j}\right)\right|>\left|z_{j}\right|^{j}$. Let $\left(z_{j}^{\prime}, w_{j}^{\prime}\right)=F\left(z_{j}, w_{j}\right)$ so that $z_{j}^{\prime}=f\left(z_{j}\right)-\delta w_{j}$ and $w_{j}^{\prime}=z_{j}$. Since $\{H=0\}$ is invariant we have that $H\left(z_{j}^{\prime}, w_{j}^{\prime}\right)=0$.

By Lemma 3.10 there exists $d \in \mathbb{N}$ such that $\left|w_{j}\right|<\left|z_{j}\right|^{d}$ for $j$ sufficiently large. Hence for $j$ sufficiently large

$$
\left|z_{j}^{\prime}\right|=\left|f\left(z_{j}\right)-\delta w_{j}\right| \geq\left|f\left(z_{j}\right)\right|-\left|\delta w_{j}\right| \geq\left|f\left(z_{j}\right)\right| / 2 .
$$

It follows that

$$
\left|p\left(w_{j}^{\prime}\right)\left(z_{j}^{\prime}\right)^{N_{1}}\right| \geq c\left|z_{j}^{\prime}\right|^{N_{1}} \geq c\left|z_{j}^{\prime}\right|^{N_{1}-1} \cdot \frac{\left|z_{j}\right|^{j}}{2},
$$


where $c>0$ is a constant. But since $z_{j}=w_{j}^{\prime}$ it follows that for large enough $j$, all terms of the form $\alpha_{k, \ell}\left(z_{j}^{\prime}\right)^{k}\left(w_{j}^{\prime}\right)^{\ell}$ for $k \leq N_{1}-1$ will be negligible compared to $p\left(w_{j}^{\prime}\right)\left(z_{j}^{\prime}\right)^{N_{1}}$, which contradicts $H\left(z_{j}^{\prime}, w_{j}^{\prime}\right)=0$.

\section{Classification of recurrent components}

In this section we only assume that $F$ is a holomorphic automorphism of $\mathbb{C}^{2}$ with constant Jacobian $\delta$.

Definition 4.1 A point $x \in \mathbb{C}^{2}$ is recurrent if its orbit $\left(F^{n}(x)\right)$ accumulates at $x$ itself. A periodic Fatou component $\Omega$ is called recurrent if there exists a point $z \in \Omega$ whose orbit $\left(F^{n}(z)\right)$ accumulates at a point $w \in \Omega$.

Since the class of holomorphic automorphism of $\mathbb{C}^{2}$ with constant Jacobian is closed under composition, by replacing $F$ with an iterate we can restrict to the case where $\Omega$ is invariant. For an invariant Fatou component $\Omega$, a limit map $h$ is a holomorphic function $h: \Omega \rightarrow \mathbb{P}^{2}$ such that $f^{n_{k}} \rightarrow h$ uniformly on compact sets of $\Omega$ for some subsequence $n_{k} \rightarrow \infty$.

Theorem 4.2 Let $F$ be a holomorphic automorphism of $\mathbb{C}^{2}$ with constant Jacobian $\delta$ and let $\Omega$ be an invariant recurrent Fatou component for $F$. Then there exists a holomorphic retraction $\rho$ from $\Omega$ to a closed complex submanifold $\Sigma \subset \Omega$, called the limit manifold, such that for all limit maps $h$ there exists an automorphism $\eta$ of $\Sigma$ such that $h=\eta \circ \rho$. Every orbit converges to $\Sigma$, and $\left.F\right|_{\Sigma}: \Sigma \rightarrow \Sigma$ is an automorphism. Moreover,

- If $\operatorname{dim} \Sigma=0$, then $\Omega$ is the basin of an attracting fixed point, and is biholomorphically equivalent to $\mathbb{C}^{2}$.

- If $\operatorname{dim} \Sigma=1$, either $\Sigma$ is biholomorphic to a circular domain A, and there exists a biholomorphism from $\Omega$ to $A \times \mathbb{C}$ which conjugates the map $F$ to

$$
(z, w) \mapsto\left(e^{i \theta} z, \frac{\delta}{e^{i \theta}} w\right),
$$

where $\theta$ is irrational, or there exists $j \in \mathbb{N}$ such that $\left.F^{j}\right|_{\Sigma}=\mathrm{id}_{\Sigma}$, and there exists a biholomorphism from $\Omega$ to $\Sigma \times \mathbb{C}$ which conjugates the map $F^{j}$ to

$$
(z, w) \mapsto\left(z, \delta^{j} w\right)
$$

- $\operatorname{dim} \Sigma=2$ if and only if $|\delta|=1$. In this case there exists a sequence of iterates converging to the identity on $\Omega$.

By a circular domain we mean either the disk, the punctured disk, an annulus, the complex plane or the punctured plane. For the polynomial Hénon maps case, see [5] and [19].

Let $\left(F^{n_{k}}\right)$ be a convergent subsequence of iterates on $\Omega$, with $F^{n_{k}}(z) \rightarrow w \in \Omega$. We denote the limit of $\left(F^{n_{k}}\right)$ by $g$. 
Lemma 4.3 The image $g(\Omega)$ is contained in $\mathbb{C}^{2}$.

Proof If there is a point $x \in \Omega$ for which $g(x)$ belongs to the line at infinity $\ell^{\infty}$, then $g(\Omega) \subset \ell^{\infty}$ (see e.g. the proof of Lemma 2.4), which gives a contradiction.

Definition 4.4 We define the maximal rank of $g$ as $\max _{p \in \Omega} \operatorname{rk}\left(d_{p} g\right)$.

\subsection{Maximal rank 0}

Lemma 4.5 Suppose that $g$ has maximal rank 0 . Then $g(\Omega)$ is the single point $w$, which is an attracting fixed point.

Proof Since the maximal rank is 0, the map $g$ is constant and must therefore equal $w$. Since $F$ and $g$ commute, the point $w$ must be fixed. Suppose that the differential $d_{w} F$ has an eigenvalue of absolute value $\geq 1$. Then the same is true for all iterates $F^{n_{k}}$. Hence they cannot converge to a constant map. So $w$ must be an attracting fixed point.

It follows that $\Omega$ is the attracting basin of the point $w$, and the entire sequence $F^{n}$ converges to $g$. In this case the limit manifold $\Sigma$ is the point $\{w\}$.

\subsection{Maximal rank 2}

Theorem 4.6 Suppose that g has maximal rank 2. Then there exists a subsequence $\left(m_{k}\right)$ so that $F^{m_{k}} \rightarrow$ Id on $\Omega$.

Proof Let $x$ be a point of maximal rank 2. There exist an open neighborhood $U$ of $x$ and an open neighborhood $V^{\prime}$ of $g(x)$ such that $g: U \rightarrow V^{\prime}$ is a biholomorphism. Denote $h:=g^{-1}$ defined on $V^{\prime}$. Let $V \subset \subset V^{\prime}$ be an open neighborhood of $g(x)$. Since $F^{n_{k}} \rightarrow g$ on $U$, we have that $V \subset F^{n_{k}}(U)$ for large $k$ and the maps $\left(F^{n_{k}}\right)^{-1}$ converge to $h$ uniformly on compact subsets of $V$. In particular $V \subset \Omega$. Replace $n_{k}$ by a subsequence so that $n_{k+1}-n_{k} \nearrow \infty$. We can then write $F^{n_{k+1}-n_{k}}=F^{n_{k+1}} \circ\left(F^{n_{k}}\right)^{-1}$ on $V$. If we set $m_{k}:=n_{k+1}-n_{k}$, then $F^{m_{k}} \rightarrow$ Id on $V$. Since we are in the Fatou set this implies that $F^{m_{k}} \rightarrow$ Id on $\Omega$.

It follows that every point $p \in \Omega$ is recurrent and that $F$ is volume preserving. The following fact is trivial but we recall it for convenience.

Lemma 4.7 Let $\left(G_{n}: \Omega \subset \mathbb{C}^{2} \rightarrow \mathbb{C}^{2}\right)$ be a sequence of injective holomorphic mappings which are volume preserving. If $G_{n}$ converges to $G$ uniformly on compact subsets, then $G$ is holomorphic, injective and volume preserving.

Proof The map $G$ is holomorphic and $d G_{n} \stackrel{n \rightarrow \infty}{\longrightarrow} d G$, and thus $G$ is volume preserving. Thus by Hurwitz Theorem $G$ is injective.

Corollary 4.8 Suppose that $g$ has maximal rank 2. Then if h is any limit map of $F$ on $\Omega$, then either $h$ is injective and $h(\Omega) \subset \Omega$ or $h(\Omega) \subset \ell^{\infty}$. 
Proof Assume that $h(\Omega) \subset \mathbb{C}^{2}$. Then by Lemma 4.7 the map $h$ is holomorphic and injective. Arguing as in the proof of Theorem 4.6 we get that $h(\Omega) \subset \Omega$.

Proposition 4.9 If $g$ has maximal rank 2 then each orbit $\left(F^{n}(z)\right)$ is contained in a compact subset of $\Omega$.

Proof Let $\left(K_{j}\right)$ be an exhaustion of $\Omega$ by compact subsets such that $K_{j} \subset \stackrel{\circ}{K}_{j+1}$ for all $j \in \mathbb{N}$. By passing to a subsequence of the exhaustion if needed, we may assume that $F\left(K_{j}\right) \subset \subset K_{j+1}$. Let $p \in \Omega$. We can assume that $p \in K_{1}$, and let $r>0$ be such that $B(p, r) \subset K_{1}$.

We may assume that if $F^{n}(p) \in K_{j}$ then $F^{n}(B(p, r)) \subset K_{j+1}$. Indeed, suppose by contradiction that there exist $j \in \mathbb{N}$ and subsequence $\ell_{k}$ such that $F^{\ell_{k}}(p) \in K_{j}$ for all $k \in \mathbb{N}$, but

$$
F^{\ell_{k}}(B(p, r)) \not \subset K_{j+k} .
$$

Then up to passing to a subsequence, the sequence $F^{\ell_{k}}$ converges uniformly on compact subsets of $\Omega$ to a map $h$ which satisfies $h(\Omega) \subset \Omega$. Hence we have $h(B(p, r)) \subset \subset \Omega$, which contradicts (7). Similarly we may assume that if $F^{n}(p) \notin$ $K_{j}$ then $F^{n}(B(p, r)) \cap K_{j-1}=\varnothing$.

Suppose by contradiction that the orbit of $p$ is not contained in a compact subset of $\Omega$. Then the orbit of $p$ is not contained in any $K_{j}$. But since $p$ is a recurrent point, the orbit of $p$ must also return to $K_{1}$ infinitely often. Thus, there exists a sequence $k_{1}<l_{1}<m_{1}<k_{2}<l_{2}<m_{2}<\cdots$ and a strictly increasing sequence $\left(n_{j}\right), n_{j} \geq 3$, such that

(i) Each $F^{k_{j}}(p)$ lies in $K_{4} \backslash K_{3}$

(ii) For $k_{j}<n<l_{j}$ the points $F^{n}(p)$ lie outside of $K_{3}$.

(iii) Each $F^{l_{j}}(p)$ lies outside $K_{n_{j}}$,

(iv) Each point $F^{m_{j}}(p)$ lies in $K_{1}$,

(v) For $k_{j}<n<m_{j}$ the points $F^{n}(p)$ lie in $K_{n_{j+1}-2}$.

We claim that the sets $F^{k_{j}}(B(p, r))$ must be pairwise disjoint. To see this, suppose that $F^{k_{i}}(B(p, r)) \cap F^{k_{j}}(B(p, r)) \neq \varnothing$ for some $i<j$. Then clearly

$$
F^{k_{i}+n}(B(p, r)) \cap F^{k_{j}+n}(B(p, r)) \neq \varnothing, \quad \forall n \in \mathbb{N} .
$$

If $l_{j}-k_{j}>m_{i}-k_{i}$, then a contradiction is obtained since $F^{k_{j}+m_{i}-k_{i}}(B(p, r)) \cap$ $K_{2}=\varnothing$ due to (ii) while $F^{m_{i}}(B(p, r)) \subset K_{2}$ due to (iv). If $l_{j}-k_{j}<m_{i}-k_{i}$, then a contradiction is obtained since $F^{l_{j}}(B(p, r)) \cap K_{n_{j}-1}=\varnothing$ due to (iii), while $F^{k_{i}+l_{j}-k_{j}}(B(p, r)) \subset K_{n_{i+1}-1} \subset K_{n_{j}-1}$ due to (v). Finally, if $l_{j}-k_{j}=m_{i}-k_{i}$, then $F^{m_{i}}(B(p, r)) \cap F^{\ell_{j}}(B(p, r)) \neq \varnothing$, which contradicts (iii) and (iv). This proves the claim. Since $F$ is volume preserving and the volume of $K_{4} \backslash K_{3}$ is finite, we have a contradiction.

Corollary 4.10 The limit of any convergent subsequence $\left(F^{n_{k}}\right)$ is an automorphism of $\Omega$. 
Proof By Corollary 4.8 and Proposition 4.9 the limit $h$ is a biholomorphic map $h$ : $\Omega \rightarrow h(\Omega) \subset \Omega$. Suppose that $p \in \Omega \backslash h(\Omega)$. Let $K$ be a compact subset of $\Omega$. Then for all large enough $k, p \in \Omega \backslash F^{n_{k}}(K)$. Hence $F^{-n_{k}}(p) \in \Omega \backslash K$. Fix such $k$. By Theorem 4.6 there exists $m>n_{k}$ so that $F^{m}$ is close enough to the identity so that $F^{m-n_{k}}(p) \in \Omega \backslash K$. This contradicts Proposition 4.9.

In this case the limit manifold $\Sigma$ is the whole $\Omega$.

Remark 4.11 It follows that the maximal rank of a limit map is independent of the chosen convergent subsequence.

\subsection{Maximal rank 1}

We now consider the case where the limit map $g$ has maximal rank 1. By Remark 4.11 every other limit of a convergent subsequence on $\Omega$ must also have maximal rank 1 .

Recall that $\left(F^{n_{k}}\right)$ is a convergent subsequence of iterates on $\Omega$ such that $F^{n_{k}}(z) \rightarrow$ $w \in \Omega$. Replace $n_{k}$ by a subsequence so that $n_{k+1}-n_{k} \nearrow \infty$. Let $\left(m_{k}\right)$ be a subsequence of $\left(n_{k+1}-n_{k}\right)$ so that $F^{m_{k}}$ converges, uniformly on compact subsets of $\Omega$. From now on we assume that $g$ is the limit of the sequence $\left(m_{k}\right)$.

Remark 4.12 Notice that $g(w)=w$. Actually, if follows by the construction that there exists an open neighborhood $N$ of $z$ in $\Omega$ such that $g(N) \subset \Omega$ and for all $y \in g(N)$, $g(y)=y$.

Lemma 4.13 The map $F$ is strictly volume decreasing.

Proof By assumption the Jacobian determinant $\delta$ of $F$ is constant. Since $\Omega$ is recurrent we have $|\delta| \leq 1$. Since $\left(F^{n_{k}}\right)$ converges to the map $g: \Omega \rightarrow \mathbb{C}^{2}$ of maximal rank 1 , it follows that $|\delta|<1$.

We write $\Sigma:=g(\Omega)$. Notice that $\Sigma$ is a subset of $\bar{\Omega} \cap \mathbb{C}^{2}$, and that, since $F$ and $g$ commute, the map $\left.F\right|_{\Sigma}: \Sigma \rightarrow \Sigma$ is bijective. We need a Lemma.

Lemma 4.14 Let $U$ be an open set in $\mathbb{C}^{2}$, and let $h: U \rightarrow \mathbb{C}^{2}$ be a holomorphic map of maximal rank 1 . Then for all $w \in h(U)$, the fiber $h^{-1}(w)$ has no isolated points.

Proof Assume by contradiction that $q \in h^{-1}(w)$ is isolated. If $\epsilon$ is small enough, then $h(\partial B(q, \epsilon))$ is disjoint from $w$. Hence there exists a small ball $B(w, \delta)$ which is disjoint from $h(\partial B(q, \epsilon))$, and if we restrict $h$ to $V:=h^{-1}(B(w, \delta)) \cap B(q, \epsilon)$, then $h: V \rightarrow B(w, \delta)$ is a proper holomorphic map. Let $\zeta \in V$ be such that $\operatorname{rk}_{\zeta} h=1$. Then the level set $h^{-1}(h(\zeta))$ contains a closed analytic curve in $V$. Such curve is not relatively compact in $V$, and this contradicts properness.

It is not clear a priori that $\Sigma$ is a complex submanifold, but we will show, following a classical normalization procedure, that there exists a smooth Riemann surface $\widehat{\widehat{\Sigma}}$ such that the self-map $F$ on $\Sigma$ can be lifted to a holomorphic automorphism $\widehat{F}$ on $\widehat{\Sigma}$. Note that such normalization procedure was used in a similar context in [31]. 
Lemma 4.15 For each point $z \in \Omega$ there is an open connected neighborhood $U(z) \subset$ $\Omega$, an affine disk $\Delta_{z} \subset \Omega$ through $z$ and an injective holomorphic mapping $\gamma_{z}: \Delta_{z} \rightarrow$ $\mathbb{C}^{2}$ such that

(1) $\gamma_{z}\left(\Delta_{z}\right)$ is an irreducible local complex analytic curve which is smooth except possibly at $\gamma_{z}(0)$ where it could have a cusp singularity,

(2) $\gamma_{z}\left(\Delta_{z}\right)=g(U(z))$.

Moreover, if $g$ has rank 1 at $z$, then $\gamma_{z}\left(\Delta_{z}\right)$ is smooth and $\gamma_{z}=\left.g\right|_{\Delta_{z}}$.

Proof If $g$ has rank 1 at $z$, the result follows immediately from the constant rank Theorem. So suppose that $g$ has rank 0 at $z$, and let $\Delta_{z} \subset \Omega$ be an affine disk through $z$ on which $g$ is not constant. By the Puiseux expansion of $g: \Delta_{z} \rightarrow \mathbb{C}^{2}$, it follows that, up to taking a smaller $\Delta_{z}, g\left(\Delta_{z}\right)$ is an irreducible local complex analytic curve (with possibly an isolated cusp singularity at $g(z)$ ). Hence $g\left(\Delta_{z}\right)$ is the zero set of a holomorphic function $G$ defined in a open neighborhood $V$ of $g(z)$. Let $U(z)$ be the connected component of $g^{-1}(V)$ containing $z$. We claim that $G \circ g$ vanishes identically on $U(z)$, which implies that $g(U(z))=g\left(\Delta_{z}\right)$. If not, then $(G \circ g)^{-1}(0)$ is a closed complex analytic curve in $U(z)$ containing $\Delta_{z}$. Pick a point $q \in \Delta_{z}$ where locally $(G \circ g)^{-1}(0)=\Delta_{z}$. Then $g^{-1}(g(q))$ is isolated at $q$ since $g$ is not constant on $\Delta_{z}$, which gives a contradiction by Lemma 4.14.

Finally, again by the Puiseux expansion of $g: \Delta_{z} \rightarrow \mathbb{C}^{2}$, there exists a holomorphic injective map $\gamma_{z}: \Delta_{z} \rightarrow \mathbb{C}^{2}$ such that $\gamma_{z}\left(\Delta_{z}\right)=g\left(\Delta_{z}\right)$.

Remark 4.16 For all $z \in \Omega$, there exists a unique surjective holomorphic map $h_{z}: U(z) \rightarrow \Delta_{z}$ such that $g=\gamma_{z} \circ h_{z}$ on the neighborhood $U(z)$. If $g$ has rank 1 at $z$, then $\left.h_{z}\right|_{\Delta_{z}}=\mathrm{id}$.

Consider the disjoint union $\bigsqcup_{z \in \Omega} \Delta_{z}$, and define an equivalence relation in the following way: $(x, z) \simeq(y, w)$ if and only if $\gamma_{z}(x)=\gamma_{w}(y)$ and the images coincide locally near this point. Define $\widehat{\Sigma}$ as $\bigsqcup_{z \in \Omega} \Delta_{z} / \simeq$, endowed with the quotient topology, and denote $\pi_{\simeq}: \bigsqcup_{z \in \Omega} \Delta_{z} \rightarrow \widehat{\Sigma}$ the projection to the quotient. It is easy to see that the map $\pi_{\simeq}$ is open. For all $z \in \Omega$, define a homeomorphism $\pi_{z}: \Delta_{z} \rightarrow \widehat{\Sigma}$ as $\pi_{z}(x):=[(x, z)]$.

Definition 4.17 We define a continuous map $\gamma: \widehat{\Sigma} \rightarrow \mathbb{C}^{2}$ such that $\gamma(\widehat{\Sigma})=\Sigma$ in the following way: $\gamma([(x, z)])=\gamma_{z}(x)$. Notice that this is well defined. The map $g: \Omega \rightarrow \mathbb{C}^{2}$ can be lifted to a unique surjective continuous map $\widehat{g}: \Omega \rightarrow \widehat{\Sigma}$ such that $g=\gamma \circ \widehat{g}$. Such map is defined on $U(z)$ as $\widehat{g}:=\pi_{z} \circ h_{z}$. Notice that if $g$ has rank 1 at $z$ then $\left.\widehat{g}\right|_{\Delta_{z}}=\pi_{z}$.

Lemma 4.18 The topological space $\widehat{\Sigma}$ is connected, second countable and Hausdorff.

Proof Since $\widehat{\Sigma}=\hat{g}(\Omega)$, and $\Omega$ is connected, it follows that $\widehat{\Sigma}$ is connected. Since $\widehat{g}$ is open, it follows also that $\widehat{\Sigma}$ is second countable. Let $[(x, z)] \neq[(y, w)] \in \widehat{\Sigma}$. Then we have two cases. Either $\gamma_{z}(x) \neq \gamma_{w}(y)$, or $\gamma_{z}(x)=\gamma_{w}(y)$ but the images do not coincide locally near this point. In both cases there exist a neighborhood $U \subset \Delta_{z}$ of $x$ and a neighborhood $V \subset \Delta_{w}$ of $y$ such that $\pi_{\simeq}(U) \cap \pi_{\simeq}(V)=\varnothing$. 
We claim that the collection of charts $\left(\pi_{z}\right)_{z \in \Omega}$ gives $\widehat{\Sigma}$ the structure of a smooth Riemann surface. Let $z, w \in \Omega$ such that $\pi_{z}\left(\Delta_{z}\right) \cap \pi_{w}\left(\Delta_{w}\right) \neq \varnothing$. Then consider the map

$$
\pi_{w}^{-1} \circ \pi_{z}: \pi_{z}^{-1}\left(\pi_{z}\left(\Delta_{z}\right) \cap \pi_{w}\left(\Delta_{w}\right)\right) \rightarrow \pi_{w}^{-1}\left(\pi_{z}\left(\Delta_{z}\right) \cap \pi_{w}\left(\Delta_{w}\right)\right) .
$$

Let $x \in \Delta_{z}, y \in \Delta_{w}$ such that $\pi_{z}(x)=\pi_{w}(y)$. This means that $\gamma_{z}(x)=\gamma_{w}(y)$ and the images coincide locally near this point. There exists an open neighborhood $U \subset \Delta_{z}$ of $x$, an open neighborhood $V \subset \Delta_{w}$ of $y$, and a unique biholomorphic function $k: U \rightarrow V$ such that $\gamma_{w} \circ k=\gamma_{z}$. It is easy to see that $k=\pi_{w}^{-1} \circ \pi_{z}$ on $U$.

Remark 4.19 With the complex structure just defined on $\widehat{\Sigma}$, the maps $\gamma$ and $\widehat{g}$ are holomorphic.

Definition 4.20 Define $R \subset \widehat{\Sigma}$ as the set of points $\zeta \in \widehat{\Sigma}$ such that there exists $z \in \Omega$ with $\widehat{g}(z)=\zeta$ and $\mathrm{rk}_{z} \widehat{g}=1$.

Lemma 4.21 The set $\widehat{\Sigma} \backslash R$ is discrete.

Proof Let $w \in \Omega$ such that $\mathrm{rk}_{w} \widehat{g}=0$. By the identity principle there exists a neighborhood $V$ of $w$ in $\Delta_{w}$ such that $\operatorname{rk}_{z}\left(\left.h_{w}\right|_{\Delta_{w}}\right)=1$ for all $z \in V \backslash\{w\}$. The result follows since $\widehat{g}=\pi_{w} \circ h_{w}$ on $U(w)$, and $\pi_{w}: \Delta_{w} \rightarrow \pi_{w}\left(\Delta_{w}\right)$ is a biholomorphism.

Lemma 4.22 There exists a unique holomorphic map $\widehat{F}: \widehat{\Sigma} \rightarrow \widehat{\Sigma}$ such that the following diagram commutes:

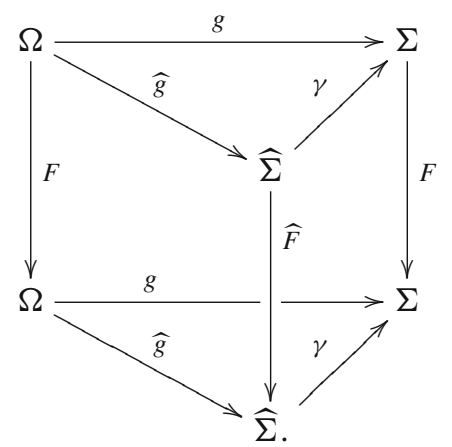

Proof Let $\zeta \in R$, and let $z \in \Omega$ such that $\widehat{g}(z)=\zeta$ and $\operatorname{rk}_{z} \widehat{g}=1$. Define on $\pi_{z}\left(\Delta_{z}\right)$ the map $\widehat{F}:=\widehat{g} \circ F \circ \pi_{z}^{-1}$. This is well-defined and holomorphic away from the discrete closed set $\widehat{\Sigma} \backslash R$, and can be extended holomorphically to the whole $\widehat{\Sigma}$.

The inverse of $\widehat{F}$ is given by $\widehat{F^{-1}}$, therefore $\widehat{F}$ is an automorphism.

Lemma 4.23 The Riemann surface $\widehat{\Sigma}$ contains an open subset on which the sequence $\left(\widehat{F}^{m_{k}}\right)$ converges to the identity. 
Proof By Remark 4.12 there exists an open neighborhood $N$ of $z$ such that $g(N) \subset \Omega$ and for all $y \in g(N), F^{m_{k}}(y) \rightarrow y$. Then, for all $y \in g(N)$,

$$
\widehat{F}^{m_{k}}(\widehat{g}(y))=\widehat{g}\left(F^{m_{k}}(y)\right) \rightarrow \widehat{g}(y) .
$$

The set $\widehat{g}(N)$ is open.

Lemma 4.24 Either there exists a $j \in \mathbb{N}$ for which $\widehat{F}^{j}=\mathrm{Id}$, or $\widehat{\Sigma}$ is biholomorphic to a circular domain, and the action of $\widehat{F}$ is conjugate to an irrational rotation.

Recall that by a circular domain we mean either the disk, the punctured disk, an annulus, the complex plane or the punctured plane.

Proof Assume that $\widehat{F}^{j} \neq$ Id for all $j \leq 1$. Since the holomorphic map $\gamma: \widehat{\Sigma} \rightarrow \mathbb{C}^{2}$ is nonconstant, it follows that the Riemann surface $\widehat{\Sigma}$ is not compact. Thus if $\widehat{\Sigma}$ is not a hyperbolic Riemann surface, then it has to be biholomorphic either to $\mathbb{C}$ or to $\mathbb{C}^{*}$. In both cases, since $\widehat{F}$ is an automorphism, it is easy to see that Lemma 4.23 implies that $\widehat{F}$ is conjugate to an irrational rotation. If $\widehat{\Sigma}$ is hyperbolic, then the family $\left(\widehat{F}^{n}\right)$ is normal, and thus Lemma 4.23 implies that the sequence $\left(\widehat{F}^{m_{k}}\right)$ converges to the identity uniformly on compact subsets of $\widehat{\Sigma}$. Thus the automorphism group of $\widehat{\Sigma}$ is non-discrete. Hence (see e.g. [16, p. 294]) the Riemann surface $\widehat{\Sigma}$ is biholomorphic to a circular domain and the action of $\widehat{F}$ is conjugate to an irrational rotation.

Definition 4.25 Define the set $I \subset \widehat{\Sigma} \times \widehat{\Sigma}$ as the set of pairs $(x, y)$ such that $x \neq y$ and $\gamma(x)=\gamma(y)$. Define the set $C \subset \widehat{\Sigma}$ as the set of $x$ such that $\gamma: \widehat{\Sigma} \rightarrow \mathbb{C}^{2}$ has rank 0 at $x$.

Since the map $\pi \simeq$ is open, it follows immediately that the set $I$ is discrete in $\widehat{\Sigma} \times \widehat{\Sigma}$ and that the set $C$ is discrete in $\widehat{\Sigma}$.

Our goal is to prove that the set $\Sigma$ is a closed complex submanifold of $\Omega$. We will first consider the case where $\widehat{\Sigma}$ is biholomorphic to a circular domain and $\widehat{F}$ is conjugate to an irrational rotation.

Lemma 4.26 If $\widehat{F}$ is conjugate to an irrational rotation then there is at most one element $\zeta_{0} \in C$. The set I is empty, and thus the map $\gamma: \widehat{\Sigma} \rightarrow \Sigma$ is injective.

Proof The set $C$ is invariant by $\widehat{F}$. Since the action of $\widehat{F}$ is conjugate to an irrational rotation, and since $C$ is discrete it follows that $C$ can only contain the center of rotation $\zeta_{0}$ (if there is one).

Similarly, the set $I$ is invariant by the map $(x, y) \mapsto(\widehat{F}(x), \widehat{F}(y))$, but this contradicts the discreteness of $I$.

Lemma 4.27 The set $C \subset \widehat{\Sigma}$ is empty.

Proof If $\widehat{\Sigma}$ has no center of rotation, then there is nothing to prove. Suppose for the purpose of a contradiction that there is a center of rotation $\zeta_{0} \in \widehat{\Sigma}$ and that $\zeta_{0} \in C$.

Then $\Sigma$ has a cusp at $z:=\gamma\left(\zeta_{0}\right)$. Notice that $F(z)=z$. Since $\widehat{F}$ acts on $\widehat{\Sigma}$ as a rotation, it follows that the tangent direction of $\Sigma$ to $z$ is an eigenvector of $d_{z} F$ with 
eigenvalue $\left|\lambda_{1}\right|=1$. Since $F$ is strictly volume decreasing, the other eigenvalue $\lambda_{2}$ of $d_{z} F$ satisfies $0<\left|\lambda_{2}\right|<1$. Thus we obtain a forward invariant cone in $T_{z}\left(\mathbb{C}^{2}\right)$, centered at the line $T_{z}(\Sigma)$. Extending this cone to a constant cone field in a neighborhood of $z$, it follows that we obtain stable manifolds through $z$ and all nearby points in $\Sigma$, giving a continuous Riemann surface foliation near the point $z$, see the reference [23] for background on normal hyperbolicity.

Since $\widehat{F}$ acts on $\widehat{\Sigma}$ as a rotation, the stable manifolds through different points in $\Sigma$ must be distinct. However, this is not possible in a neighborhood of $z$. To see this, let $h$ be a locally defined holomorphic function such that $\Sigma$ equals the zero set of $h$ near $z$. We may assume that $h$ vanishes to higher order only at $z$. Now consider the restriction of $h$ to the stable manifold through $z$. Then $h$ has a multiple zero at $z$, hence by Rouché's Theorem, the number of zeroes for nearby stable manifolds is also greater than one. But since nearby stable manifolds are transverse to $\Sigma$, and $h$ does not vanish to higher order in nearby points, it follows that the restriction of $h$ to nearby stable manifolds must have multiple single zeroes. Hence these nearby stable manifolds intersect $\Sigma$ in more than one point, giving a contradiction.

Let $\widehat{V} \subset \subset \widehat{\Sigma}$ be open and invariant under the action of $\widehat{F}$, and let $V$ be its image in $\mathbb{C}^{2}$, which is an embedded complex submanifold of $\mathbb{C}^{2}$.

We claim that there exists a continuous function $\varphi: V \rightarrow(0, \infty)$, bounded from above and from below by compactness, such that

$$
\left\|d_{z}\left(\left.F\right|_{V}\right)\right\|=\frac{\varphi(z)}{\varphi(F(z))} .
$$

Indeed, regardless of whether $\widehat{\Sigma}$ is a hyperbolic or Euclidean Riemann surface, there exists a conformal metric $\|\cdot\|$ on $\widehat{\Sigma}$ which is invariant under $\widehat{F}$. The function

$$
\varphi(z):=\left\|d_{z} \gamma^{-1}\right\|
$$

satisfies (8).

Given $\epsilon>0$ and $z \in V$, we define the tangent cone $\mathcal{C}_{z} \subset T_{z}\left(\mathbb{C}^{2}\right)$ by

$$
\mathcal{C}_{z}=\left\{w:\left|\left\langle w, v_{z}^{\perp}\right\rangle\right| \leq \epsilon \varphi(z)^{2}\left|\left\langle w, v_{z}\right\rangle\right|\right\}
$$

where $v_{z}$ is a unit tangent vector to $z \in V$ and $v_{z}^{\perp}$ is a unit vector orthogonal to $v_{z}$.

Lemma 4.28 One can choose $\epsilon>0$ sufficiently small so that

$$
d_{z} F\left(\mathcal{C}_{z}\right) \subset \subset \mathcal{C}_{F(z)}
$$

Proof This is a matter of linear algebra. Without loss of generality we may assume that $v_{z}=v_{F(z)}=(1,0)$. Thus, the cone field $\mathcal{C}_{z}$ contains all vectors $w=\left(w_{1}, w_{2}\right)$ for which

$$
\left|w_{2}\right| \leq \epsilon \varphi(z)^{2}\left|w_{1}\right|
$$


The vector $d_{z} F(w)$ is given by

$$
d_{z} F(w)=\left(\theta(z) w_{1}+\alpha(z) w_{2}, \beta(z) w_{2}\right),
$$

where $|\theta(z)|=\frac{\varphi(z)}{\varphi(F(z))}$. Since $F$ has constant Jacobian determinant $\delta$ it follows that

$$
|\theta(z) \beta(z)|=|\delta|
$$

Since $|\alpha(z)|$ is bounded on $V$, by choosing $\epsilon$ sufficiently small we can guarantee that for all $z \in V$ we have

$$
\frac{1-|\delta|}{\varphi(F(z)) \varphi(z)}>\epsilon|\alpha(z)|,
$$

from which it follows that

$$
\epsilon \varphi(F(z))^{2}\left|d_{z} F(w)_{1}\right|>\left|d_{z} F(w)_{2}\right|
$$

Thus, $d_{z} F$ sends the cone $\mathcal{C}_{z}$ strictly into $\mathcal{C}_{F(z)}$.

Lemma 4.29 The image $\Sigma$ is contained in $\Omega$.

Proof Since $F$ is $C^{1}$, we can extend the invariant cone field to a neighborhood $\mathcal{N}(V)$. Let $z$ be a point whose forward orbit remains in $\mathcal{N}(V)$, which holds in particular for all points in $V$. Then there exists a stable manifold $W^{s}(z)$, transverse to $V$, and these stable manifolds fill up a neighborhood of $V$.

The forward iterates of $\mathrm{F}$ form a normal family on this neighborhood, which implies that $V$ is contained in the Fatou set.

Corollary 4.30 The map $g: \Omega \rightarrow \Sigma$ is a holomorphic retraction. In particular $\Sigma$ is a closed smooth one-dimensional embedded submanifold.

Proof On $\Sigma=g(\Omega) \subset \Omega$,

$$
g=\lim _{k \rightarrow \infty} F^{m_{k}}=\mathrm{Id}
$$

which proves that $g$ is a holomorphic retraction.

In the case where $\widehat{F}^{j}$ equals the identity, it follows that $F^{j}$ equals the identity on $\Sigma$. The next lemma, together with the fact that the Jacobian $\delta$ of $F$ satisfies $|\delta|<1$, shows that there cannot be cusps and self-intersections in this case.

Lemma 4.31 If a local biholomorphic map $G$ of a neighborhood of the origin in $\mathbb{C}^{2}$ is the identity on a complex analytic curve $\gamma$ with any kind of singularity at the origin, then $d_{0} G$ is the identity.

Proof The curve $\gamma$ will be the zero set of a holomorphic function $\phi$ which vanishes generically to first order on the curve, but vanishes to at least order 2 at the origin. Then we write $G(z, w)=(z+A(z, w) \phi, w+B(z, w) \phi)$, which gives the result. 
We immediately get stable manifolds transverse to $\Sigma$, which imply as above that $g: \Omega \rightarrow \Sigma$ is a holomorphic retraction.

Lemma 4.32 The retraction $g$ has constant rank 1 on $\Omega$.

Proof Since $g$ is a retraction to the 1-dimensional manifold $\Sigma$, there exists a neighborhood $V$ of $\Sigma$ such that $\operatorname{rk}_{x} g=1$ for all $x \in V$. Let $x \in \Omega$ and $N \geq 0$ be such that $y=F^{N}(x) \in V$. The result follows from the fact that $F^{N}$ has rank 2 and that

$$
g \circ F^{N}(x)=F^{N} \circ g(x) .
$$

Remark 4.33 The stable manifolds of the points in $\Sigma$ fill up a neighborhood of $\Sigma$. Since all orbits in the Fatou component $\Omega$ get close to $\Sigma$, this implies that the stable manifolds of the points in $\Sigma$ fill up the whole $\Omega$. Thus for any limit map $h: \Omega \rightarrow \mathbb{P}^{2}$ we have that $h(\Omega) \subset \Sigma$. Moreover, by Lemma 4.24, the restriction $\left.h\right|_{\Sigma}$ is an automorphism of $\Sigma$, and thus $h(\Omega)=\Sigma$. Notice that for all $z \in \Sigma$ the fiber $g^{-1}(z)$ coincides with the stable manifold $W^{s}(z)$. Hence $h=\left.h\right|_{\Sigma} \circ g$.

Remark 4.34 In the view of the contents of this section if a Fatou component $\Omega$ is recurrent then for every $z \in \Omega$ the orbit is relatively compact in $\Omega$.

Now we investigate the complex structure of $\Omega$. Notice that $\Sigma$, being an open Riemann surface, is Stein.

Proposition 4.35 If some iterate $\left.F^{j}\right|_{\Sigma}$ is the identity, then there exists a biholomorphism $\Psi: \Omega \rightarrow \Sigma \times \mathbb{C}$ which conjugates the map $F^{j}$ to $(z, w) \mapsto\left(z, \delta^{j} w\right)$.

Proof Let $L$ be the holomorphic line bundle on $\Sigma$ given by $\operatorname{Ker} d g$ restricted to $\Sigma$. Since $\Sigma$ is Stein, there exists a neighborhood $U$ of $\Sigma$ and an injective holomorphic map $h: U \rightarrow L$ such that for all $z \in \Sigma$ we have that $h(z)=0_{z}$, and $h$ maps the fiber $g^{-1}(z) \cap U$ biholomorphically into a neighborhood of $0_{z}$ in the fiber $L_{z}$ [21, Proposition 3.3.2]. We can assume that $\left.d_{z} h\right|_{L_{z}}=\mathrm{id}_{L_{z}}$. Notice that the map $\left.d_{z} F^{j}\right|_{L_{z}}: L_{z} \rightarrow L_{z}$ acts as multiplication by $\delta^{j}$. The sequence $\left(\left.d F\right|_{L} ^{-n j} \circ h \circ F^{n j}\right)$ is eventually defined on compact subsets of $\Omega$ and converges uniformly on compact subsets to a biholomorphism $\Psi: \Omega \rightarrow L$, conjugating $F^{j}$ to $\left.d F^{j}\right|_{L}: L \rightarrow L$. The line bundle $L$ is holomorphically trivial since $\Sigma$ is a Stein Riemann surface.

If no iterate of $F$ is the identity on $\Sigma$, then $\Sigma$ is a biholomorphic to a circular domain $A$, and by the same proof as in [5, Proposition 6] there exists a biholomorphism from $\Omega$ to $A \times \mathbb{C}$ which conjugates the map $F$ to

$$
(z, w) \mapsto\left(e^{i \theta} z, \frac{\delta}{e^{i \theta}} w\right)
$$




\subsection{Transcendental Hénon maps}

So far in this section we have not used that the maps we study here are of the form $F(z, w)=(f(z)-\delta w, z)$. Absent of any further assumptions we do not know how to use this special form to obtain a more precise description of recurrent Fatou components. We do however have the following consequence of Proposition 3.2 and Theorem 4.2.

Corollary 4.36 Let $F$ be a transcendental Hénon map, and assume that $f$ has order of growth strictly smaller than $\frac{1}{2}$. Then any recurrent periodic Fatou component $\Omega$ of rank 1 must be the attracting basin of a Riemann surface $\Sigma \subset \Omega$ which is biholomorphic to a circular domain, and $f$ acts on $\Sigma$ as an irrational rotation.

\section{Baker domain}

A Baker domain for a transcendental function $f$ in $\mathbb{C}$ is a periodic Fatou component on which the iterates converge to the point $\infty$, an essential singularity for $f$. Notice that, in contrast, every polynomial $p$ in $\mathbb{C}$ can be extended to a rational function of $\mathbb{P}^{1}$ for which $\infty$ is a super-attracting fixed point. The first example of a Baker domain was given by Fatou [17], who considered the function

$$
f(z)=z+1+e^{-z}
$$

and showed that the right half-plane $\mathbb{H}$ is contained in an invariant Baker domain.

For holomorphic automorphisms of $\mathbb{C}^{2}$ the definition of essential singularity at infinity has to be adapted in order to be meaningful.

Definition 5.1 Let $F$ be a holomorphic automorphism of $\mathbb{C}^{2}$. We call a point $[p: q$ : $0] \in \ell^{\infty}$ an essential singularity at infinity if for all $[s: t: 0] \in \ell^{\infty}$ there exists a sequence $\left(z_{n}, w_{n}\right)$ of points in $\mathbb{C}^{2}$ converging to $[p: q: 0]$ whose images $F\left(z_{n}, w_{n}\right)$ converge to $[s: t: 0]$.

Remark 5.2 If $F$ is a transcendental Hénon map, then any point $[p: q: 0]$ in $\ell_{\infty}$ is an essential singularity at infinity. To see this, it is in fact sufficient to consider only points on the line $\{(p \zeta, q \zeta)\}$ when $p \neq 0$. Define the entire function

$$
g(\zeta)=\frac{f(p \zeta)-f(0)-\delta q \zeta}{p \zeta} .
$$

Since $f$ is transcendental, the function $g$ must also be transcendental. Let $\left(\zeta_{n}\right)$ be a sequence of points in $\mathbb{C}$ converging to $\infty$ for which $g\left(\zeta_{n}\right) \rightarrow \frac{s}{t}$. Then

$$
F\left(p \zeta_{n}, q \zeta_{n}\right)=\left(p \zeta_{n} \cdot g\left(\zeta_{n}\right)+f(0), p \zeta_{n}\right) \rightarrow[s: t: 0] .
$$

Here we consider the iteration of a map on $\mathbb{C}^{2}$ analogous to (9), namely the transcendental Hénon map

$$
F(z, w):=\left(e^{-z}+2 z-w, z\right)
$$


and we show that it admits an invariant Fatou component $U$ on which the iterates tend to the point $[1: 1: 0]$ on the line at infinity.

Remark 5.3 Notice that by Remark 5.2 the point $[1: 1: 0]$ is an essential singularity at infinity for the map $F$, and this implies that $F$ cannot be extended, even continuously, to the point $[1: 1: 0]$. The situation is radically different for a polynomial Hénon map $H$, for which the escaping set

$$
I_{\infty}:=\left\{(z, w) \in \mathbb{C}^{2}:\left\|H^{n}(z, w)\right\| \rightarrow \infty\right\}
$$

is a Fatou component on which all orbits converge to the point $[1: 0: 0]$, and the map $H$ extends to a holomorphic self-map $\widehat{H}$ of $\mathbb{P}^{2} \backslash\{[0: 1: 0]\}$ with a super-attracting fixed point at [1:0:0].

For a transcendental function $f$ in $\mathbb{C}$ it is known that Baker domains are simply connected (proper) domains of $\mathbb{C}$ and by results of Cowen [11] (see also [9, Lemma 2.1]) the function $f$ is semi-conjugate to one of the following automorphisms:

(1) $z \mapsto \lambda z \in \operatorname{Aut}(\mathbb{H})$, where $\lambda>1$,

(2) $z \mapsto z \pm i \in \operatorname{Aut}(\mathbb{H})$,

(3) $z \mapsto z+1 \in \operatorname{Aut}(\mathbb{C})$.

In our case we show that on the Fatou component $U$ the map $F$ is conjugate to the linear map $L \in \operatorname{Aut}(\{\operatorname{Re}(z-w)>0\})$ given by

$$
L(z, w):=(2 z-w, z) .
$$

We show that the conjugacy maps $U$ onto the domain $\{\operatorname{Re}(z-w)>0\}$, proving that $U$ is biholomorphic to $\mathbb{H} \times \mathbb{C}$.

We begin by constructing an appropriate forward invariant domain $R$. For each $\alpha>0$ we define the domain

$$
R_{\alpha}:=\left\{(z, w): \operatorname{Re} z>\operatorname{Re} w+\alpha+\eta_{\alpha}(\operatorname{Re} w)\right\}
$$

where

$$
\eta_{\alpha}(x):=\frac{e^{-\alpha}}{1-e^{-\alpha}} \cdot e^{-x}:=A_{\alpha} \cdot e^{-x} .
$$

Notice that $\eta_{\alpha}>0, \frac{A_{\alpha}}{1+A_{\alpha}}=e^{-\alpha}$, and that the domains $R_{\alpha}$ are not nested.

Lemma 5.4 Each domain $R_{\alpha}$ is forward invariant.

Proof Let $\left(z_{0}, w_{0}\right) \in R_{\alpha}$. We claim that $\operatorname{Re} z_{1}>\operatorname{Re} w_{1}+\alpha+\eta_{\alpha}\left(\operatorname{Re} w_{1}\right)$. Since $w_{1}=z_{0}$,

$$
\begin{aligned}
\operatorname{Re} z_{1}-\operatorname{Re} w_{1} & =\operatorname{Re} e^{-z_{0}}+\operatorname{Re} z_{0}-\operatorname{Re} w_{0}>\alpha+\eta_{\alpha}\left(\operatorname{Re} w_{0}\right)+\operatorname{Re}\left(e^{-z_{0}}\right) \\
& \geq \alpha+\eta_{\alpha}\left(\operatorname{Re} w_{0}\right)-e^{-\operatorname{Re} z_{0}}
\end{aligned}
$$


Hence the claim follows if we show that

$$
\eta_{\alpha}\left(\operatorname{Re} w_{0}\right) \geq \eta_{\alpha}\left(\operatorname{Re} z_{0}\right)+e^{-\operatorname{Re} z_{0}}
$$

This is the same as $A_{\alpha} e^{-\operatorname{Re} w_{0}} \geq\left(1+A_{\alpha}\right) e^{-\operatorname{Re} z_{0}}$, or equivalently $e^{-\alpha} \geq e^{-\operatorname{Re} z_{0}+\operatorname{Re} w_{0}}$. The latter is satisfied because $\operatorname{Re} z_{0}-\operatorname{Re} w_{0} \geq \alpha$ (since $\eta_{\alpha}$ is always positive).

We immediately obtain the following.

\section{Corollary 5.5 The domain}

$$
R:=\bigcup_{\alpha>0} R_{\alpha}
$$

is forward invariant.

Remark 5.6 Let $\left(z_{0}, w_{0}\right) \in R_{\alpha}$. Since $w_{1}=z_{0}$ we have that

$$
\operatorname{Re} z_{1}-\operatorname{Re} z_{0}>\alpha+\eta_{\alpha}\left(\operatorname{Re} z_{0}\right)
$$

It easily follows that then $\operatorname{Re} z_{n}>\operatorname{Re} z_{0}+n \alpha$, and that $\operatorname{Re} w_{n}>\operatorname{Re} z_{0}+(n-1) \alpha$.

Lemma 5.7 All the orbits in $R$ converge uniformly on compact subsets to the point [1: $1: 0]$ on the line at infinity.

Proof Let $K$ be a compact subset of $R_{\alpha}$ for some $\alpha>0$. Let $M>0$. Then by Remark 5.6 we have that $\left|z_{n}\right|$ and $\left|w_{n}\right|$ converge to $\infty$ uniformly on $K$. Moreover, since for all $n \geq 0$ we have that $z_{n+1}-w_{n+1}=z_{n}-w_{n}+e^{-z_{n}}$, it follows that

$$
z_{n}-w_{n}=z_{0}-w_{0}+\sum_{j=0}^{n-1} e^{-z_{j}}
$$

and thus $\frac{z_{n}}{w_{n}}$ converges to 1 uniformly on $K$.

The domain $R$ is therefore contained in an invariant Fatou component $U$. Our next goal is to show that $R$ is an absorbing domain for $U$, i.e.

$$
U=A:=\bigcup_{n \in \mathbb{N}} F^{-n}(R) .
$$

It is immediate that $A$ is contained in $U$. In order to show that $U$ is not larger than $A$, we will for the first time use the plurisubharmonic method referred to in the introduction.

Definition 5.8 Define the sequence of pluriharmonic functions $\left(u_{n}: U \rightarrow \mathbb{R}\right)_{n \geq 1}$ by

$$
u_{n}\left(z_{0}, w_{0}\right):=\frac{-\operatorname{Re}\left(z_{n}\right)}{n} .
$$


Lemma 5.9 The functions $u_{n}$ are uniformly bounded from above on compact subsets of $U$, and

$$
\limsup _{n \rightarrow+\infty} u_{n} \leq 0
$$

Proof Let $K$ be a compact subset of $U$. Since $U$ is a Fatou component, on $K$ the orbits converge uniformly to $[1: 1: 0]$. So for every $\epsilon>0$ there exists $n_{\epsilon} \geq 0$ such that

$$
\left|\frac{z_{n}}{z_{n-1}}\right|=\left|\frac{z_{n}}{w_{n}}\right|<1+\epsilon,
$$

for all $n>n_{\epsilon}$ and for all $\left(z_{0}, w_{0}\right) \in K$. It follows that for every $\epsilon>0$ there exists $C=C_{\epsilon}$ such that

$$
\max \left\{\left|z_{n}\right|,\left|w_{n}\right|\right\} \leq C \cdot(1+\epsilon)^{n}
$$

for every $n \in \mathbb{N}$ and for all $\left(z_{0}, w_{0}\right) \in K$. Let $\beta>0$. We claim that there exists an $N>0$ so that $u_{n}<\beta$ on $K$ for every $n \geq N$. To prove this claim, let us suppose by contradiction that there exist a sequence $\left(z_{0}^{k}, w_{0}^{k}\right)_{k \in \mathbb{N}}$ in $K$ and a strictly increasing sequence $(n(k))_{k \in \mathbb{N}}$ in $\mathbb{N}$ such that

$$
u_{n(k)}\left(z_{0}^{k}, w_{0}^{k}\right) \geq \beta
$$

for all $k \in \mathbb{N}$. Then $\operatorname{Re}\left(z_{n(k)}^{k}\right) \leq-n(k) \cdot \beta$. It follows that

$$
\begin{aligned}
\left|z_{n(k)+1}^{k}\right| & =\left|e^{-z_{n(k)}^{k}}+2 z_{n(k)}^{k}-w_{n(k)}^{k}\right| \\
& \geq\left|e^{-z_{n(k)}^{k}}\right|-\left|2 z_{n(k)}^{k}-w_{n(k)}^{k}\right| \\
& \geq e^{n(k) \cdot \beta}-3 C(1+\epsilon)^{n(k)}
\end{aligned}
$$

By taking $\epsilon>0$ sufficiently small so that $e^{\beta}>(1+\epsilon)$, we obtain, for $k$ sufficiently large,

$$
\left|z_{n(k)+1}^{k}\right|>C(1+\epsilon)^{n(k)+1}
$$

which contradicts (12).

The claim implies that there is a uniform bound from above for the functions $u_{n}$ on $K$, and that

$$
\limsup _{n \rightarrow \infty} u_{n} \leq 0
$$

which completes the proof. 
Lemma 5.10 Let $H$ be a compact subset of $A$. Then there exists $\gamma>0$ such that on $H$,

$$
\limsup u_{n} \leq-\gamma
$$

Proof Let $\left(z_{0}, w_{0}\right) \in R_{\alpha}$ for some $\alpha>0$. Then by Remark 5.6 we have,

$$
u_{n}\left(z_{0}, w_{0}\right)<-\frac{\operatorname{Re} z_{0}}{n}-\alpha, \quad \forall n \geq 1,
$$

which implies that $\lim \sup _{n \rightarrow+\infty} u_{n}\left(z_{0}, w_{0}\right) \leq-\alpha$.

Let now $H$ be a compact subset in $A$. Then there exist $\alpha_{1}, \ldots, \alpha_{k}>0$ and $n_{1}, \ldots, n_{k} \in \mathbb{N}$ such that

$$
H \subset F^{-n_{1}}\left(R_{\alpha_{1}}\right) \cup \cdots \cup F^{-n_{k}}\left(R_{\alpha_{k}}\right) .
$$

Thus, on $H$,

$$
\limsup _{n \rightarrow+\infty} u_{n} \leq \max \left\{-\alpha_{1}, \ldots,-\alpha_{k}\right\}
$$

Lemma 5.11 On $U \backslash A$, $\lim \sup _{n \rightarrow+\infty} u_{n}=0$.

Proof Let $\left(z_{0}, w_{0}\right) \in U \backslash A$. Suppose by contradiction that there exists $\alpha>0$ and $N \in \mathbb{N}$ such that $u_{n}\left(z_{0}, w_{0}\right) \leq-\alpha$ for all $n \geq N$, that is,

$$
\frac{\operatorname{Re} z_{n}}{n} \geq \alpha, \quad \forall n \geq N
$$

Let $n_{0} \geq 0$ be such that $\eta_{\alpha / 3}\left(\operatorname{Re} z_{n}\right)<\alpha / 3$ for all $n>n_{0}$. For all $0<\beta<\alpha$ there are arbitrarily large $n$ such that $\operatorname{Re} z_{n+1}-\operatorname{Re} z_{n} \geq \beta$. Setting $\beta:=2 \alpha / 3$, there exists $n \geq n_{0}$ such that

$$
\operatorname{Re} z_{n+1}-\operatorname{Re} z_{n}>2 \alpha / 3>\alpha / 3+\eta_{\alpha / 3}\left(\operatorname{Re} z_{n}\right)
$$

which implies that $\left(z_{n+1}, w_{n+1}\right) \in R_{\alpha / 3}$.

Proposition 5.12 The set $R$ is an absorbing domain.

Proof Let us assume, for the purpose of a contradiction, that $U \neq A$. Define

$$
u(z):=\limsup _{n \rightarrow+\infty} u_{n}(z)
$$

and let $u^{\star}$ be its upper semicontinuous regularization. Then by [25, Prop 2.9.17] the function $u^{\star}$ is plurisubharmonic. By Lemmas 5.10 and 5.11 the function $u^{\star}$ is strictly negative on $A$, and constantly equal to zero on $U \backslash A$. But then $u^{\star}$ contradicts the sub-mean value property at boundary points $\zeta \in \partial A$. 
Remark 5.13 It is easy to see that for all $n \in \mathbb{Z},(z, w) \in \mathbb{C}^{2}$,

$$
L^{n}(z, w)=((n+1) z-n w, n z-(n-1) w) .
$$

Definition 5.14 We denote by $\Omega$ the domain $\left\{(z, w) \in \mathbb{C}^{2}: \operatorname{Re}(z-w)>0\right\}$, which is biholomorphic to $\mathbb{H} \times \mathbb{C}$.

Theorem 5.15 There exists a biholomorphism $\psi: U \rightarrow \Omega$ which conjugates $F$ to the map L. In particular $U$ is biholomorphic to $\mathbb{H} \times \mathbb{C}$.

Proof We will construct the map $\psi$ as the uniform limit on compact subsets of $U$ of the maps

$$
\psi_{n}:=L^{-n} \circ F^{n}: U \rightarrow \mathbb{C}^{2}
$$

Notice that for all $n \geq 1$, the mapping $\psi_{n}$ is an injective volume-preserving holomorphic mapping. We have

$$
\begin{aligned}
\delta_{n}\left(z_{0}, w_{0}\right) & :=\left\|L^{-n-1}\left(F^{n+1}\left(z_{0}, w_{0}\right)\right)-L^{-n}\left(F^{n}\left(z_{0}, w_{0}\right)\right)\right\| \\
& =\left\|L^{-n}\left(L^{-1} F\left(z_{n}, w_{n}\right)-\left(z_{n}, w_{n}\right)\right)\right\| \\
& =\left\|L^{-n}\left(0,-e^{-z_{n}}\right)\right\| \\
& =\left\|\left(-n e^{-z_{n}},(-n-1) e^{-z_{n}}\right)\right\| \leq \sqrt{2}(n+1) e^{-\operatorname{Re} z_{n}} .
\end{aligned}
$$

By Remark 5.6, on $R_{\alpha}$

$$
\sqrt{2}(n+1) e^{-\operatorname{Re} z_{n}} \leq \sqrt{2}(n+1) e^{-\operatorname{Re} z_{0}-n \alpha},
$$

Hence for all $\left(z_{0}, w_{0}\right) \in R_{\alpha}$, we have $\sum_{k=0}^{\infty} \delta_{n}\left(z_{0}, w_{0}\right)<+\infty$, and the sequence $\left(\psi_{n}\right)_{n \geq 0}$ converges uniformly on compact subsets of $R$ to an injective volumepreserving (see Lemma 4.7) holomorphic mapping $\psi: R \rightarrow \mathbb{C}^{2}$, satisfying

$$
L \circ \psi=\psi \circ F
$$

Since $R$ is an absorbing domain, $\psi$ extends to an injective volume-preserving holomorphic mapping (still denoted by $\psi$ ) defined on the whole Fatou component $U$ and still satisfying (13). We claim that $\psi(U)=\Omega$.

We first show that $\psi(U) \subset \Omega$. Let $\left(z_{0}, w_{0}\right)$ be in $R_{\alpha}$. Notice that the Euclidean distance $d\left(R_{\alpha}, \Omega^{\complement}\right)>0$. We claim that

$$
\lim _{n \rightarrow \infty}\left\|\psi\left(z_{n}, w_{n}\right)-\left(z_{n}, w_{n}\right)\right\|=0 .
$$


Indeed,

$$
\begin{aligned}
\left\|\psi\left(z_{n}, w_{n}\right)-\left(z_{n}, w_{n}\right)\right\| & \leq \sum_{j=0}^{\infty}\left\|\psi_{j+1}\left(z_{n}, w_{n}\right)-\psi_{j}\left(z_{n}, w_{n}\right)\right\| \\
& =\sum_{j=0}^{\infty} \delta_{j}\left(z_{n}, w_{n}\right) \leq \sqrt{2} \sum_{j=0}^{\infty}(j+1) e^{-\operatorname{Re}\left(z_{n+j}\right)}
\end{aligned}
$$

and the claim follows since $\operatorname{Re} z_{n+j} \geq \operatorname{Re} z_{0}+(n+j) \alpha$. Hence, if $n$ is large enough, $\psi\left(z_{n}, w_{n}\right) \in \Omega$. Since $\Omega$ is completely invariant under $L$ and $U$ is completely invariant under $F$, it follows that $\psi(U) \subset \Omega$.

What is left is to show that $\Omega \subset \psi(U)$. Let $\left(x_{0}, y_{0}\right) \in \Omega$, and write $\left(x_{n}, y_{n}\right)$ $:=L^{n}\left(x_{0}, y_{0}\right)$. By definition of $\Omega$ we have that $\beta:=\operatorname{Re}\left(x_{0}-y_{0}\right)>0$. Note that $\operatorname{Re}\left(x_{n}-y_{n}\right)$ also equals $\beta$, and that $\operatorname{Re}\left(x_{n}\right), \operatorname{Re}\left(y_{n}\right) \rightarrow \infty$. Let $0<\alpha<\beta$. Recalling that $\eta_{\alpha}(x) \rightarrow 0$ as $x \rightarrow+\infty$ it follows that $\left(x_{n}, y_{n}\right) \in R_{\alpha}$ for all $n \in \mathbb{N}$ sufficiently large. In fact, there exists an $r>0$ and an $N \in \mathbb{N}$ such that $R_{\alpha}$ contains the closed ball $\bar{B}\left(\left(x_{n}, y_{n}\right), r\right)$ for all $n \geq N$. We claim that

$$
\lim _{n \rightarrow \infty}\|\psi-\operatorname{Id}\|_{\bar{B}\left(\left(x_{n}, y_{n}\right), r\right)}=0 .
$$

Indeed, for all $n \in \mathbb{N}$,

$$
\begin{aligned}
\|\psi-\mathrm{Id}\|_{\bar{B}\left(\left(x_{n}, y_{n}\right), r\right)} & \leq \sum_{j=0}^{\infty}\left\|\psi_{j+1}-\psi_{j}\right\|_{\bar{B}\left(\left(x_{n}, y_{n}\right), r\right)} \\
& =\sum_{j=0}^{\infty}\left\|\delta_{j}\right\|_{\bar{B}\left(\left(x_{n}, y_{n}\right), r\right)} \\
& \leq \sqrt{2} \sum_{j=0}^{\infty}(j+1)\left\|e^{-\operatorname{Re}\left(\pi_{1} \circ F^{j}\right)}\right\|_{\bar{B}\left(\left(x_{n}, y_{n}\right), r\right)}
\end{aligned}
$$

Assume now that $n \geq N$. Since $\bar{B}\left(\left(x_{n}, y_{n}\right), r\right) \in R_{\alpha}$ we have that for all $(x, y)$ $\in \bar{B}\left(\left(x_{n}, y_{n}\right), r\right)$,

$$
e^{-\operatorname{Re} \pi_{1}\left(F^{j}(x, y)\right)} \leq e^{-\operatorname{Re} x-j \alpha} \leq e^{-\operatorname{Re} x_{0}-(n+j) \alpha+r},
$$

where the last inequality follows from the fact that for all $n \in \mathbb{N}$ and $(x, y)$ $\in \bar{B}\left(\left(x_{n}, y_{n}\right), r\right)$ we have $\operatorname{Re} x \geq \operatorname{Re} x_{0}+n \alpha-r$. This proves the claim.

Let $n \geq N$ be such that $\|\psi-\mathrm{Id}\| \leq \frac{r}{2}$ on $\bar{B}\left(\left(x_{n}, y_{n}\right), r\right)$. By Rouché's Theorem in several complex variables it follows that $\left(x_{n}, y_{n}\right) \in \psi\left(\bar{B}\left(\left(x_{n}, y_{n}\right), r\right)\right) \subset \psi(U)$. Since $\Omega$ is completely invariant under $L$ and $U$ is completely invariant under $F$, it follow that $\left(x_{0}, y_{0}\right) \in \psi(U)$. 


\section{Escaping wandering domain}

Definition 6.1 Let $F$ be a transcendental Hénon map. A Fatou component $\Omega$ is a wandering domain if it is not preperiodic. A wandering domain

(1) is escaping if all orbits converge to the line at infinity,

(2) is oscillating if there exists an unbounded orbit and an orbit with a bounded subsequence,

(3) is orbitally bounded if every orbit is bounded.

For polynomials in $\mathbb{C}$ it is known that wandering domains cannot exist [30]. For transcendental functions there are examples of escaping wandering domains. [7] uses for example the function $f(z)=z+\lambda \sin (2 \pi z)+1$ for suitable $\lambda$. There are also examples of oscillating wandering domains $[10,15]$, and it is an open question whether orbitally bounded wandering domains can exist.

It follows from the existence of the filtration that a polynomial Hénon map does not admit any escaping or oscillating wandering domain. In the remainder of the paper we will give examples of both escaping and oscillating wandering domains for transcendental Hénon maps. The existence of orbitally bounded wandering domains is an open question for both polynomial and transcendental Hénon maps.

We start with the escaping case, and we will be inspired by the construction of escaping wandering domains for transcendental functions. Similar to [7] we will use functions of the form

$$
\tilde{f}(z)=z+\sin (2 \pi z)+\lambda
$$

with appropriate values of $\lambda$. It will be convenient for us to take the constant $\lambda$ such that we obtain an escaping orbit, all consisting of critical points. Note that

$$
\tilde{f}^{\prime}(z)=1+2 \pi \cos (2 \pi z)
$$

The critical points of $\tilde{f}$ are therefore the points that satisfy

$$
\cos (2 \pi z)=\frac{-1}{2 \pi}
$$

A computation gives that $\tilde{f}$ has two distinct bi-infinite sequences of critical points

$$
\begin{array}{ll}
c_{n,+}:=\alpha+n & \text { with } \sin \left(2 \pi c_{n,+}\right)=+\sqrt{1-\frac{1}{4 \pi^{2}}} \\
c_{n,-}:=-\alpha+n & \text { with } \sin \left(2 \pi c_{n,-}\right)=-\sqrt{1-\frac{1}{4 \pi^{2}}}
\end{array}
$$

for the appropriate value of $\frac{1}{4}<\alpha<\frac{1}{2}$ given by solving (14). 
Note that the set of critical points is invariant under translation by 1 . By taking

$$
\lambda=1-\sqrt{1-\frac{1}{4 \pi^{2}}},
$$

$\tilde{f}$ acts as a translation by 1 on the sequence $c_{n,+}$.

For simplicity of notation we consider the map $f$ obtained by conjugating $\tilde{f}$ with a real translation by $\alpha$, so that the critical points $c_{n,+}$ for $\tilde{f}$ are mapped to critical points $z_{n}=n$ for $f$. Thus, $f$ will act on $\mathbb{Z}$ as a translation by 1 , and $f$ commutes with translation by 1 .

Consider the function $g(z):=f(z)-1$, which commutes with $f$ and with the translation by 1 . For the function $g$ each point $z_{n}$ is a super-attracting fixed point. For all $n \in \mathbb{Z}$ denote by $B_{n}$ the immediate basin for $g$ of the point $z_{n}$. Clearly the basins $B_{n}$ are disjoint, $B_{n+1}=B_{n}+1$, and $f\left(B_{n}\right) \subset B_{n+1}$. If one shows that each $B_{n}$ is also a Fatou component for $f$, then clearly each $B_{n}$ is an escaping wandering domain for $f$.

There are two classical ways in one dimensional complex dynamics to show that each $z_{n}$ belongs to a different Fatou component for such a function $f$. One is by constructing curves in the Julia set that separate the points $z_{n}$ [12, p. 183], and the other one is by showing that any two commuting maps which differ by a translation have the same Julia set [2, Lemma 4.5]. The first kind of argument is typically unavailable in higher dimensions. The proof we present is an ad hoc version of the second argument. We will define transcendental Hénon maps $F, G: \mathbb{C}^{2} \rightarrow \mathbb{C}^{2}$ which behave similarly to $f, g$, then use $G$ to show that the norms of the differentials $d G^{n}$ and $d F^{n}$ at points on the boundaries of the attracting basins for $G$ grow exponentially in $n$, and finally use the latter information to imply that those boundaries must be contained in the Julia set for $F$. Since the attracting basins are disjoint for $G$, the corresponding sets are disjoint for $F$, giving a sequence of wandering domains.

Let us define the Hénon map

$$
F(z, w)=(f(z)+\delta(z-1)-\delta w, z)
$$

where $f$ is as constructed before and $\delta>0$ is some constant to be suitably chosen later. Since $f$ commutes with translation by 1 , the map $F$ commutes with translation by $(1,1)$. Moreover, on the sequence of points $(n, n-1)$ the map $F$ acts as a translation by $(1,1)$. We also define the map

$$
G(z, w):=F(z, w)-(1,1)
$$

Hence the points $P_{n}=(n, n-1)$ are all fixed points of $G$. Since the points $z_{n}$ are critical points of $f$, we can choose $\delta$ sufficiently small so that the fixed points $P_{n}=(n, n-1)$ are attracting for $G$. Denote by $A_{n}$ the attracting basin of the point $P_{n}$, which is biholomorphic to $\mathbb{C}^{2}$. It is easy to see that $A_{n+1}=A_{n}+(1,1)$ and that $F\left(A_{n}\right)=A_{n+1}$. We claim that each $A_{n}$ is also a Fatou component for $F$, and thus each $\left(A_{n}\right)$ is an escaping wandering domain. We first need a Lemma. 
Lemma 6.2 Let $0<\lambda<1$, and let $\left(\xi_{n}: \mathbb{D} \rightarrow \mathbb{C}\right)$ be a sequence of holomorphic functions satisfying

$$
\left|\xi_{n}(z)\right|<\lambda^{n}
$$

for all $z \in \mathbb{D}$. Let $0<\beta<1$. Then

$$
\left|\xi_{n}^{(k)}(0)\right|<k ! \cdot \beta^{k}
$$

for

$$
k<\frac{n}{\log _{\lambda}(\beta)}
$$

Proof It follows from the Cauchy estimates since the assumption on $k$ is equivalent to

$$
\lambda^{n}<\beta^{k}
$$

Theorem 6.3 Let $F$ be a transcendental Hénon map that commutes with a translation $T=T_{(1,1)}$. Write

$$
G:=T^{-1} \circ F
$$

assume that $G$ has an attracting fixed point $Q$, and denote its basin of attraction by A. Then $A$ is also a Fatou component of $F$.

Proof Notice that $\left(T^{k}(Q)\right)$ is an $F$-orbit converging to the point $[1: 1: 0]$ on the line at infinity. Since $F^{n}=T^{n} \circ G^{n}$ for all $n \in \mathbb{N}$, it follows that the $F$-orbits of all points in $A$ converge to [1: $1: 0]$. Hence $A$ is contained in a Fatou component $\Omega$ of $F$. We show that $\Omega$ equals $A$ by proving that all boundary points of $A$ are contained in the Julia set of $F$.

Without loss of generality we may assume that the point $Q$ is the origin. Let $P \in \partial A$. Since $P \notin A$, its $G$-orbit avoids some definite ball centered at $(0,0)$, hence there exists $0<\mu<1$ for which

$$
\left\|G^{n}(P)\right\| \geq \sum_{k=0}^{\infty} \mu^{k}
$$

for all $n \in \mathbb{N}$. For each $n$ we can choose an orthogonal projection $\pi_{n}$ onto a complex line through $(0,0)$ so that

$$
\left|\pi_{n}\left(G^{n}(P)\right)\right| \geq \sum_{k=0}^{\infty} \mu^{k} .
$$


Let $\varphi: \mathbb{C} \rightarrow \mathbb{C}^{2}$ be an affine embedding for which $P \in \varphi(\mathbb{D})$ and $\varphi(0) \in A$. We will show that for any choice of $\varphi$ the derivatives of $G^{n} \circ \varphi$ grow exponentially fast for some point in the disk $D(0, \eta)$, where $\eta>\frac{1}{\mu}$ is chosen independently of $\varphi$. This will imply that some point in $\varphi(D(0, \eta))$ is contained in the Julia set of $F$. Since $\varphi$ is arbitrary, one can choose $\varphi(D(0, \eta))$ to be contained in arbitrarily small neighborhoods of $P$, giving a sequence of points $Z_{n} \rightarrow P$ belonging to the Julia set of $F$. Since the latter is closed, the statement of the theorem follows.

We consider the sequence of maps $\psi_{n}: \mathbb{C} \rightarrow \mathbb{C}$ defined by

$$
\psi_{n}:=\pi_{n} \circ G^{n} \circ \varphi .
$$

Let $r>0$ be such that $\varphi(D(0, r)) \subset \subset A$. Then there exist $C>0$ and $\lambda<1$ so that

$$
\left\|\psi_{n}\right\|_{D(0, r)}<C \cdot \lambda^{n}
$$

By instead defining

$$
\psi_{n}=\pi_{n} \circ G^{n+N} \circ \varphi .
$$

for some large integer $N \in \mathbb{N}$ we may assume that

$$
\left\|\psi_{n}\right\|_{D(0, r)}<\lambda^{n}
$$

By defining the maps $\xi_{n}(z):=\psi_{n}(r \cdot z)$ we obtain a sequence of maps $\left(\xi_{n}: \mathbb{D} \rightarrow \mathbb{C}\right)$ that satisfy the conditions of Lemma 6.2 , which we apply with $\beta:=\mu \cdot r$. Hence

$$
\left|\xi_{n}^{(k)}(0)\right|<k ! \mu^{k} r^{k} \quad \text { for } k<\frac{n}{\log _{\lambda}(\mu r)},
$$

which implies that

$$
\left|\psi_{n}^{(k)}(0)\right|<k ! \mu^{k} \quad \text { for } k<\frac{n}{\log _{\lambda}(\mu r)} .
$$

Writing $\zeta=\varphi^{-1}(P) \in \mathbb{D}$, we also have that

$$
\left|\psi_{n}(\zeta)\right| \geq \sum_{k=0}^{\infty} \mu^{k}
$$

for all $n$. Writing

$$
\psi_{n}(z)=\sum_{k=0}^{\infty} a_{k} z^{k}
$$

by (16) there is at least one $k \in \mathbb{N}$ for which

$$
\left|a_{k}\right| \geq \mu^{k}
$$


and by (15), $k \geq \frac{n}{\log _{\lambda}(\mu r)}$. Putting things together we get

$$
\left|\psi_{n}^{(k)}(0)\right| \geq k ! \mu^{k} \quad \text { for some } k \geq \frac{n}{\log _{\lambda}(\mu r)} .
$$

Let $\eta>\frac{1}{\mu}$. By Cauchy estimates this implies the existence of a $z_{*} \in D(0, \eta)$ for which

$$
\left|\psi_{n}^{\prime}\left(z_{*}\right)\right|>\frac{\eta^{k-1} \mu^{k} k !}{(k-1) !} \geq C \Lambda^{n}
$$

where $\Lambda=(\eta \mu)^{\frac{1}{\log _{\lambda}(\mu r)}}>1$ and $C>0$ is constant. Denote $P_{*}:=\varphi\left(z_{*}\right)$. Since $\left\|\pi_{n}\right\|=1$ we get, up to changing $C$,

$$
\left\|d_{P_{*}}\left(G^{n}\right)\right\| \geq C \Lambda^{n} .
$$

It follows from $F^{n}=T^{n} \circ G^{n}$ that

$$
\left\|d_{P_{*}}\left(F^{n}\right)\right\| \geq C \Lambda^{n}
$$

We now show that a point in $\varphi(D(0, \eta))$ is contained in the Julia set of $F$. Assume for the purpose of a contradiction that $\varphi(D(0, \eta))$ is contained in the Fatou component $\Omega$. It follows that $F^{n} \rightarrow[1: 1: 0]$ uniformly on a neighborhood $U$ of $P_{*}$.

Consider the affine chart of $\mathbb{P}^{2}$ defined as $[z: w: t] \mapsto(w / z, t / z)$ defined on $\{z \neq 0\}$. On $\{z \neq 0\} \cap\{t \neq 0\}$ such chart has the expression $H(z, w)=(w / z, 1 / z)$. The sequence $\left(H \circ F^{n}: U \rightarrow \mathbb{C}^{2}\right.$ ) is defined for $n$ large enough and converges uniformly to the point $(1,0) \in \mathbb{C}^{2}$. Hence, denoting $P_{*}:=\left(z_{0}, w_{0}\right)$,

$$
d_{\left(z_{n}, w_{n}\right)} H \circ d_{\left(z_{0}, w_{0}\right)}\left(F^{n}\right)=d_{\left(z_{0}, w_{0}\right)}\left(H \circ F^{n}\right) \rightarrow 0 .
$$

We have that

$$
\left(d_{\left(z_{n}, w_{n}\right)} H\right)^{-1}=\left(\begin{array}{cc}
0 & -z_{n}^{2} \\
z_{n} & -w_{n} z_{n}
\end{array}\right) .
$$

Since $\left(z_{n}, w_{n}\right) \rightarrow[1: 1: 0]$, arguing as in (12) we obtain that for every $\epsilon>0$ there exists $C^{\prime}>0$ such that

$$
\frac{1}{\left\|\left(d_{\left(z_{n}, w_{n}\right)} H\right)^{-1}\right\|} \geq \frac{1}{C^{\prime}} \frac{1}{(1+\epsilon)^{n}}
$$

and thus

$$
\left\|d_{\left(z_{n}, w_{n}\right)} H \circ d_{\left(z_{0}, w_{0}\right)}\left(F^{n}\right)\right\| \geq \frac{\left\|d_{\left(z_{0}, w_{0}\right)}\left(F^{n}\right)\right\|}{\left\|\left(d_{\left(z_{n}, w_{n}\right)} H\right)^{-1}\right\|} \geq \frac{C}{C^{\prime}} \frac{\Lambda^{n}}{(1+\epsilon)^{n}} .
$$

Hence if $\epsilon$ is small enough, this contradicts (18). 


\section{Oscillating wandering domain}

We show in this section the existence of a transcendental Hénon map with an oscillating wandering domain biholomorphic to $\mathbb{C}^{2}$.

Notice that, up to a linear change of variables, any transcendental Hénon map can be written in the alternative form $F(z, w)=(f(z)+a w, a z)$, where $f$ is a transcendental function and $a \neq 0$. We will consider maps of the form

$$
F(z, w):=(f(z)+a w, a z), \quad f(z):=b z+O\left(|z|^{2}\right),
$$

where $a, b \in \mathbb{R}, a<1$, are such that the origin is a saddle point. For example, since the eigenvalues of $d_{0} F$ are

$$
\lambda=\frac{b \pm \sqrt{b^{2}+4 a^{2}}}{2},
$$

we can pick $a=1 / 2$, and $b=1$ to obtain $\lambda_{s}=\frac{1-\sqrt{2}}{2}, \lambda_{u}=\frac{1+\sqrt{2}}{2}$.

We will construct $F$ as the uniform limit on compact subsets of a sequence of automorphisms $F_{k}(z, w):=\left(f_{k}(z)+a w, a z\right)$ with the same value for $a$ and $b$. We note that for transcendental Hénon maps $F(z, w)=(f(z)+a w, a z), G(z, w)$ $=(g(z)+a w, a z)$ and any subset $A \subset \mathbb{C}$ we have

$$
\|f-g\|_{A}=\|F-G\|_{A \times \mathbb{C}} .
$$

\section{Proposition 7.1 There exists a sequence of entire maps}

$$
F_{k}(z, w)=\left(f_{k}(z)+a w, a z\right), \quad f_{k}(z)=b z+O\left(|z|^{2}\right), \quad k=0,1,2, \ldots
$$

a sequence of points $P_{n}=\left(z_{n}, w_{n}\right)$, where $n=0,1,2, \ldots$, sequences $R_{k} \rightarrow \infty$, $0<\epsilon_{k} \leq \frac{1}{2^{k}}$ and $\beta_{n} \rightarrow 0$, a decreasing sequence $\theta_{k} \rightarrow 0$, and strictly increasing sequences of integers $\left\{n_{k}\right\},\left\{n_{k}^{\prime}\right\}$ with $n_{k}<n_{k}^{\prime}<n_{k+1}$, such that the following five properties are satisfied:

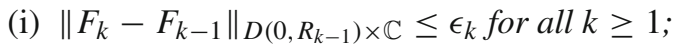

(ii) $F_{k}\left(P_{n}\right)=P_{n+1}$ for all $0 \leq n<n_{k}$;

(iii) $F_{k}\left(B\left(P_{n}, \beta_{n}\right)\right) \subset \subset B\left(P_{n+1}, \beta_{n+1}\right)$ for all $0 \leq n<n_{k}$, where $\beta_{n} \leq \frac{\theta_{\rho}}{2}$ for all $0 \leq n \leq n_{k}$

(iv) $\left|z_{n}\right|<R_{k}-\theta_{\rho}$ for all $0 \leq n<n_{k}$, and $\left|z_{n_{k}}\right|>R_{k}+5 \theta_{k}$;

(v) $P_{j} \in B\left(0, \frac{1}{k}\right)$ for some $j$ with $n_{k-1} \leq j \leq n_{k}$, for all $k \geq 1$.

Here $\rho:=\rho(n)$ denotes the unique integer for which $n_{\rho-1}^{\prime} \leq n<n_{\rho}^{\prime}$, using $n_{-1}^{\prime}=0$.

Before proving this proposition, let us show that it implies the existence of a wandering Fatou component. By (i), the maps $F_{k}$ converge uniformly on compact subsets to a holomorphic automorphism $F(z, w)=(f(z)+a w, a z)$. By (ii), (iv) and (v) it follows that $\left(P_{n}\right)$ is an oscillating orbit for $F$, that is, it is unbounded and it admits a subsequence converging to the origin. Since by (iii) for all $j \in \mathbb{N}$ the iterate images 
of $B\left(P_{j}, \beta_{j}\right)$ have uniformly bounded Euclidean diameter (in fact, the diameter goes to zero), each ball $B\left(P_{j}, \beta_{j}\right)$ is contained in the Fatou set of $F$.

Lemma 7.2 If $i \neq j$, then $P_{i}$ and $P_{j}$ are in different Fatou components of $F$, and hence $F$ has an oscillating wandering domain.

Proof For all $j \in \mathbb{N}$, denote $\Omega_{j}$ the Fatou component containing $B\left(P_{j}, \beta_{j}\right)$. Since $\beta_{n} \rightarrow 0$ as $n \rightarrow \infty$, by (iii) and by identity principle it follows that all limit functions on each $\Omega_{j}$ are constants.

Assume $j>i$ and let $k=j-i$. Let $\mathcal{N}(0)$ be a neighborhood of the origin that contains no periodic points of order less than or equal to $k$. Since the orbit $\left(P_{n}\right)$ enters and leaves a compact subset of $\mathcal{N}(0)$ infinitely often, there must be a subsequence $\left(n_{j}\right)$ for which $P_{n_{j}}$ converges to a point $z \in \mathcal{N}(0) \backslash\{0\}$. But then $P_{n_{j}+k}$ converges to $F^{k}(z) \neq z$, which implies that $P_{i}$ and $P_{j}$ cannot be contained in the same Fatou component.

We will prove Proposition 7.1 by induction on $k$. We start the induction by letting $R_{0}:=1, \theta_{0}:=1, \beta_{0}:=\frac{1}{2}$ and $P_{0}:=\left(z_{0}, w_{0}\right)$ with $\left|z_{0}\right|>6$. We set $F_{0}(z, w)$ $:=(b z+a w, a z)$ and $n_{0}:=0$.

Let us now suppose that (i)-(v) hold for certain $k$, and let us proceed to define $F_{k+1}$ and the points $\left(P_{n}\right)_{n_{k}<n \leq n_{k+1}}$. This is done in two steps. The first step relies upon the classical Lambda Lemma 7.3:

Lemma 7.3 (Lambda Lemma, see e.g. [27]) Let $G$ be a holomorphic automorphism of $\mathbb{C}^{2}$ with a saddle fixed point at the origin. Denote by $W^{s}(0)$ and $W^{u}(0)$ the stable and unstable manifolds respectively. Let $p \in W^{s}(0) \backslash\{0\}$ and $q \in W^{u}(0) \backslash\{0\}$, and let $D(p)$ and $D(q)$ be holomorphic disks through $p$ and $q$, respectively transverse to $W^{s}(0)$ and $W^{u}(0)$. Let $\epsilon>0$. Then there exists $N \in \mathbb{N}$ and $N_{1}>2 N+1$, and a point $x \in D(p)$ with $G^{N_{1}}(x) \in D(q)$ such that $\left\|G^{n}(x)-G^{n}(p)\right\|<\epsilon$ and $\left\|G^{N_{1}-n}(x)-G^{-n}(q)\right\|<\epsilon$ for $0 \leq n \leq N$, and $\left\|G^{n}(x)\right\|<\epsilon$ when $N<n<N_{1}-N$.

Using the Lambda Lemma we find a finite $F_{k}$-orbit $\left(Q_{j}\right)_{0 \leq j \leq M}$, the new oscillation, which goes inward along the stable manifold of $F_{k}$, reaching the ball $B\left(0, \frac{1}{k+1}\right)$, and then outwards along the unstable manifold of $F_{k}$.

The second step of the proof relies upon another classical result:

Lemma 7.4 (Runge approximation) Let $K \subset \mathbb{C}$ be a polynomially convex compact subset (recall that $K$ is polynomially convex if and only if $\mathbb{C} \backslash K$ is connected). Let $h \in \mathcal{O}(K)$, and let $\left\{p_{i}\right\}_{0 \leq i \leq q}$ be a set of points in $K$. Then for all $\epsilon>0$ there exists an entire holomorphic function $f \in \mathcal{O}(\mathbb{C})$ such that

$$
\|f-h\|_{K} \leq \epsilon
$$

and such that

$$
f\left(p_{i}\right)=h\left(p_{i}\right), f^{\prime}\left(p_{i}\right)=h^{\prime}\left(p_{i}\right) \quad \forall 0 \leq i \leq q .
$$


Using Runge approximation we find a map $F_{k+1}$ connecting the previously constructed finite orbit $\left(P_{n}\right)_{0 \leq n \leq n_{k}}$ with the new oscillation $\left(Q_{j}\right)_{0 \leq j \leq M}$ via a finite orbit along which $F_{k+1}$ is sufficiently contracting. The contraction neutralizes possible expansion along the new oscillation $\left(Q_{j}\right)_{0 \leq j \leq M}$, and we refer to this connecting orbit as the contracting detour.

\subsection{Finding the new oscillation}

Lemma 7.5 There exist a finite $F_{k}$-orbit $\left(Q_{j}\right):=\left(z_{j}^{\prime}, w_{j}^{\prime}\right)_{0 \leq j \leq M}$ intersecting the ball $B\left(0, \frac{1}{k+1}\right)$ and a small enough $\theta_{k+1}>0$ such that the three disks

$$
\bar{D}\left(z_{n_{k}}, \theta_{k}\right), \quad \bar{D}\left(w_{0}^{\prime} / a, \theta_{k+1}\right), \quad \bar{D}\left(z_{M}^{\prime}, \theta_{k+1}\right)
$$

are pairwise disjoint, and disjoint from the polynomially convex set

$$
K:=\bar{D}\left(0, R_{k}\right) \cup \bigcup_{0 \leq i<M} \bar{D}\left(z_{i}^{\prime}, \theta_{k+1}\right) .
$$

Proof Let $\left(\varphi^{s}, \psi^{s}\right): \mathbb{C} \rightarrow W_{F_{k}}^{s}(0,0)$ be linearizing coordinates for the stable manifold of the map $F_{k}$. Let $\zeta \in \mathbb{C}$ be a point with minimal absolute value such that

$$
\left|\psi^{s}(\zeta)\right|=a\left(\left|z_{n_{k}}\right|-4 \theta_{k}\right)
$$

It is easy to see that

$$
\left|\psi^{s}(\eta)\right|<\left|\psi^{s}(\zeta)\right|, \quad \forall \eta:|\eta|<|\zeta| .
$$

Setting $\left(u_{0}, v_{0}\right):=\left(\varphi^{s}(\zeta), \psi^{s}(\zeta)\right)$, by definition we have

$$
\frac{\left|v_{0}\right|}{a}=\left|z_{n_{k}}\right|-4 \theta_{k}
$$

Moreover, the forward orbit $\left(u_{i}, v_{i}\right)$ satisfies

$$
\left|v_{i}\right|=\left|\psi^{s}\left(\lambda_{s}^{i} \cdot \zeta\right)\right|<\left|v_{0}\right|, \quad \forall i \geq 1
$$

and since $\left|u_{i}\right|=\frac{\left|v_{i+1}\right|}{a}$ it follows that

$$
\left|u_{i}\right|<\left|z_{n_{k}}\right|-4 \theta_{k}, \quad \forall i \geq 0
$$

Similarly, we find a point $\left(s_{0}, t_{0}\right)$ in the unstable manifold $W_{F_{k}}^{u}(0,0)$ whose backwards orbit $\left(s_{-i}, t_{-i}\right)$ satisfies $\left|s_{-i}\right|<\left|s_{0}\right|$ and for which

$$
\left|s_{0}\right|=\left|z_{n_{k}}\right|-2 \theta_{k}
$$

By taking an arbitrarily small perturbation of $\left(s_{0}, t_{0}\right)$, we can make sure that the discrete sequence $\left(s_{i}\right)$ avoids the value $\frac{v_{0}}{a}$. 
Fig. 2 The first coordinates of the orbit $\left(P_{n}\right)$

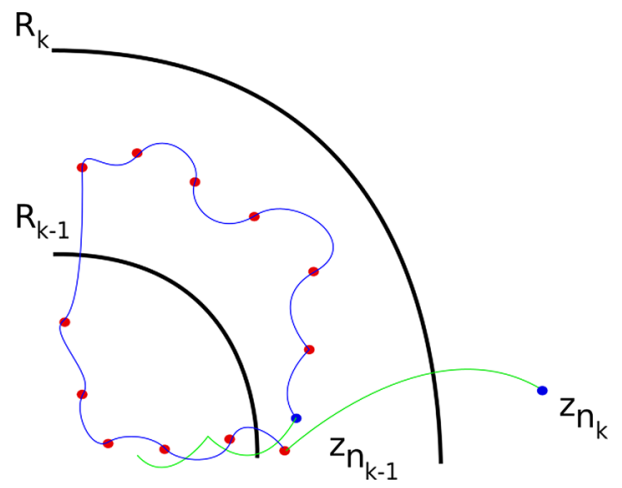

Consider arbitrarily small disks $D_{1}$ centered at $\left(u_{0}, v_{0}\right)$ and $D_{2}$ centered at $\left(s_{0}, t_{0}\right)$ transverse to the stable respectively the unstable manifold. It follows from the Lambda Lemma 7.3 that there exists a finite $F_{k}$-orbit $\left(Q_{j}\right):=\left(z_{j}^{\prime}, w_{j}^{\prime}\right)_{0 \leq j \leq M}$ which intersects the ball $B\left(0, \frac{1}{k+1}\right)$ with $Q_{0} \in D_{1}$ and $Q_{M} \in D_{2}$. If all the perturbations are chosen small enough, then the sequence $\left(z_{j}^{\prime}\right)_{0 \leq j \leq M}$ avoids the disk $\bar{D}\left(z_{n_{k}}, \theta_{k}\right)$ and the point $\frac{w_{0}^{\prime}}{a}$. Setting $\theta_{k+1}>0$ small enough completes the proof (Fig. 2).

\subsection{Connecting the orbits via the contracting detour}

By continuity of $F_{k}$ there exist constants $0<\tilde{\beta}_{j} \leq \frac{\theta_{k+1}}{2}$ for all $0 \leq j \leq M$ such that

$$
F_{k}\left(B\left(Q_{j}, \tilde{\beta}_{j}\right)\right) \subset \subset F_{k}\left(B\left(Q_{j+1}, \tilde{\beta}_{j+1}\right)\right), \quad 0 \leq j<M .
$$

To control the contraction we will need the following Lemma, a direct consequence of the formula of transcendental Hénon maps.

Lemma 7.6 Let $f \in \mathcal{O}(\mathbb{C})$ and let $F(z, w):=(f(z)+a w, a z)$. Choose $a^{\prime}$, $a^{\prime \prime}$ such that

$$
0<a^{\prime \prime}<a<a^{\prime}<1
$$

and let $D_{1} \subset \subset D_{2} \subset \mathbb{C}$ two open disks. Then there exists $\alpha>0$ such that if we have $\|f-s\|_{\bar{D}_{2}} \leq \alpha$, for some constant $s \in \mathbb{C}$, then

$$
\begin{aligned}
a^{\prime \prime}\left\|(z, w)-\left(z^{\prime}, w^{\prime}\right)\right\| & \leq\left\|F(z, w)-F\left(z^{\prime}, w^{\prime}\right)\right\| \\
& \leq a^{\prime}\left\|(z, w)-\left(z^{\prime}, w^{\prime}\right)\right\|, \quad \forall z, z^{\prime} \in \bar{D}_{1} .
\end{aligned}
$$

Fix $0<a^{\prime \prime}<a<a^{\prime}<c<1$ and let $N>0$ be such that

$$
c^{N} \cdot \beta_{n_{k}}<\tilde{\beta}_{0}
$$


We now construct the contracting detour, starting at $P_{n_{k}}$ and ending at $Q_{0}$, obtaining a contraction by at least the factor $c^{N}$. Choose a family of points $\left\{z_{j}^{\prime \prime}\right\}_{0 \leq j \leq N} \subset \mathbb{C}$ such that

(1) $z_{0}^{\prime \prime}=z_{n_{k}}, z_{N}^{\prime \prime}=\frac{w_{0}^{\prime}}{a}$,

(2) $\left|z_{j}^{\prime \prime}\right|>\left|z_{n_{k}}\right|+2$ for all $1 \leq j \leq N-1$,

(3) $\left|z_{j}^{\prime \prime}-z_{i}^{\prime \prime}\right|>2$, for all $0 \leq i \neq j \leq N-1$.

Let $R_{k+1}>0$ be such that

$$
R_{k+1}>\left|z_{j}^{\prime \prime}\right|+\theta_{k}, \forall 0 \leq j \leq N, \quad \text { and } \quad R_{k+1}>\left|z_{j}^{\prime}\right|+\theta_{k+1}, \quad \forall 0 \leq j \leq M .
$$

Define $w_{0}^{\prime \prime}:=w_{n_{k}}$ and $w_{j}^{\prime \prime}:=a z_{j-1}^{\prime \prime}$ for all $1 \leq j \leq N$, and consider the points $\left\{T_{j}\right\}_{1 \leq j \leq N} \subset \mathbb{C}^{2}$ defined as

$$
T_{j}:=\left(z_{j}^{\prime \prime}, w_{j}^{\prime \prime}\right)
$$

By the choice of the points $\left(z_{j}^{\prime \prime}\right)$ and by Lemma 7.5 it follows that the disks

$$
\left(\bar{D}\left(z_{j}^{\prime \prime}, \theta_{k}\right)\right)_{0 \leq j<N}, \quad \bar{D}\left(w_{0}^{\prime} / a, \theta_{k+1}\right), \quad \bar{D}\left(z_{M}^{\prime}, \theta_{k+1}\right)
$$

are pairwise disjoint, and disjoint from the polynomially convex set $K$. Let $H$ denote the union of such disks.

We define a holomorphic function on the polynomially convex set $K \cup H$ in the following way:

(1) $h$ coincides with $f_{k}$ on $K$,

(2) $\left.h\right|_{\bar{D}\left(z_{j}^{\prime \prime}, \theta_{k}\right)}$ is constantly equal to $z_{j+1}^{\prime \prime}-a w_{j}^{\prime \prime}$ for all $0 \leq j<N$,

(3) $\left.h\right|_{\bar{D}\left(w_{0}^{\prime} / a, \theta_{k+1}\right)}$ is constantly equal to $z_{0}^{\prime}-a w_{N}^{\prime \prime}$,

(4) $\left.h\right|_{\bar{D}\left(z_{M}^{\prime}, \theta_{k+1}\right)}$ is constantly equal to some value $A>R_{k+1}+5 \theta_{k+1}$.

By the Runge approximation Lemma 7.4 there exists a function $f_{k+1} \in \mathcal{O}(\mathbb{C})$ such that

(1) $f_{k+1}(0)=h(0)=0, f_{k+1}^{\prime}(0)=h^{\prime}(0)=b$,

(2) $f_{k+1}\left(z_{j}\right)=h\left(z_{j}\right)$ for all $0 \leq j<n_{k}$,

(3) $f_{k+1}\left(z_{j}^{\prime}\right)=h\left(z_{j}^{\prime}\right)$ for all $0 \leq j \leq M$,

(4) $f_{k+1}\left(z_{j}^{\prime \prime}\right)=h\left(z_{j}^{\prime \prime}\right)$ for all $0 \leq j \leq N$,

(5) $\left\|f_{k+1}-h\right\|_{K \cup H}<\epsilon_{k+1}$.

where $\epsilon_{k+1} \leq \frac{1}{2^{k+1}}$, and $\epsilon_{k+1}$ is also smaller than the minimum of the constants $\alpha$ given by Lemma 7.6 for the following pairs of disks:

(1) $D\left(z_{j}^{\prime \prime}, \frac{\theta_{k}}{2}\right) \subset \subset D\left(z_{j}^{\prime \prime}, \theta_{k}\right)$ for all $0 \leq j<N$,

(2) $\bar{D}\left(w_{0}^{\prime} / a, \frac{\theta_{k+1}}{2}\right) \subset \subset \bar{D}\left(w_{0}^{\prime} / a, \theta_{k+1}\right)$,

(3) $\bar{D}\left(z_{M}^{\prime}, \frac{\theta_{k+1}}{2}\right) \subset \subset \bar{D}\left(z_{M}^{\prime}, \theta_{k+1}\right)$. 
Define $F_{k+1}(z, w):=\left(f_{k+1}(z)+a w, a z\right)$. It is easy to see that the sequences of points

$$
\left(P_{n}\right)_{0 \leq n \leq n_{k}},\left(T_{j}\right)_{1 \leq j \leq N},\left(Q_{j}\right)_{0 \leq j \leq M}
$$

form an $F_{k+1}$-orbit, that is, the contracting detour $\left(T_{j}\right)_{1 \leq j \leq N}$ connects the old and new pieces of orbit.

Set

$$
n_{k}^{\prime}:=n_{k}+N+1, \quad n_{k+1}:=n_{k}^{\prime}+M+1,
$$

so that $P_{n_{k}^{\prime}}:=Q_{0}$, and $P_{n_{k+1}}:=F_{k+1}\left(Q_{M}\right)$. Define

$$
\begin{aligned}
& \beta_{n_{k}+\ell}:=\beta_{n_{k}} \cdot c^{\ell}, \quad \forall 0 \leq \ell \leq N, \\
& \beta_{n_{k}^{\prime}+\ell}:=\tilde{\beta}_{\ell}, \quad \forall 0 \leq \ell \leq M .
\end{aligned}
$$

It is easy to see that, up to taking a smaller $\epsilon_{k+1}$, the map $F_{k+1}$ satisfies property (iii). Property (iv) follows from (21) and from $A>R_{k+1}+5 \theta_{k+1}$. Since by construction the new piece of orbit $\left(Q_{j}\right)$ intersects the ball $B\left(0, \frac{1}{k+1}\right)$, property (v) is satisfied. Thus Proposition 7.1 is proved, completing the proof of the existence of an oscillating wandering domain.

\subsection{Complex structure}

We will now prove that, by making the contracting detours sufficiently long, the oscillating wandering domains can be guaranteed to be biholomorphically equivalent to $\mathbb{C}^{2}$. We denote the Fatou component containing $P_{0}$ by $\Omega$.

We have constructed an orbit $\left(P_{n}\right)$ and a sequence of radii $\left(\beta_{n}\right)$ such that

$$
F\left(B\left(P_{n}, \beta_{n}\right)\right) \subset \subset B\left(P_{n+1}, \beta_{n+1}\right) .
$$

Define the calibrated basin

$$
\Omega_{\left(P_{n}\right),\left(\beta_{n}\right)}:=\bigcup_{n \in \mathbb{N}} F^{-n}\left(B\left(P_{n}, \beta_{n}\right)\right),
$$

and notice that it is contained in $\Omega$.

Lemma 7.7 We can guarantee that the calibrated basin $\Omega_{\left(P_{n}\right),\left(\beta_{n}\right)}$ is biholomorphic to $\mathbb{C}^{2}$.

Proof For all $n \geq 0$, let $H_{n} \in \operatorname{Aut}\left(\mathbb{C}^{2}\right)$ be defined by $H_{n}(z):=P_{n}+\beta_{n} \cdot z$. For all $m \geq n \geq 0$, define

$$
\tilde{F}_{m, n}:=H_{m}^{-1} \circ F^{m-n} \circ H_{n}
$$


Then for all $n \geq 0$, we have that $\tilde{F}_{n+1, n}\left(\mathbb{B}^{2}\right) \subset \mathbb{B}^{2}$ and $\tilde{F}_{n+1, n}(0)=0$. It is easy to see that the calibrated basin $\Omega_{\left(P_{n}\right),\left(\beta_{n}\right)}$ is biholomorphic to the set $\tilde{\Omega}:=\bigcup_{n \in \mathbb{N}} \tilde{F}_{n, 0}^{-1}\left(\mathbb{B}^{2}\right)$.

If $n \in \mathbb{N}$ belongs to a contracting detour, then

$$
\frac{a^{\prime \prime}}{c}\|x\| \leq\left\|\tilde{F}_{n+1, n}(x)\right\| \leq \frac{a^{\prime}}{c}\|x\|, \quad \forall x \in \mathbb{B}^{2},
$$

where we can assume that $a^{\prime 2}<a^{\prime \prime}$. If this was the case for every $n$ then it would follow immediately that the maps

$$
\Phi_{n}:=\left(d_{0} \tilde{F}_{0, n}\right)^{-1} \circ \tilde{F}_{0, n}
$$

converge to a biholomorphism from the calibrated basin to $\mathbb{C}^{2}$, see for example [33].

While we cannot guarantee that equation (22) with $a^{\prime 2}<a^{\prime \prime}$ holds outside of the contracting detours, by making the contracting detours sufficiently long we can still ensure that $\Phi_{n}$ converges uniformly on compact subsets to a biholomorphism $\Phi: \tilde{\Omega} \rightarrow \mathbb{C}^{2}$, compare for example Theorem 1.3 of [29].

We will now show, again by using the plurisubharmonic method, that the wandering Fatou component $\Omega$ can be forced to be equal to the calibrated basin. Consider the sequence of plurisubharmonic functions $g_{n}$ on $\mathbb{C}^{2}$ given by

$$
g_{n}(z, w):=\frac{\log \left\|F^{n}(z, w)-P_{n}\right\|}{n} .
$$

Lemma 7.8 We can guarantee that the functions $g_{n}$ converge to $\log$ a on $\Omega_{\left(P_{n}\right),\left(\beta_{n}\right)} \backslash$ $\left\{P_{0}\right\}$.

Proof Take sufficiently many contractions that are sufficiently close to multiplication by $a$.

Recall from induction hypothesis (v) in Proposition 7.1 that for every $k$ there exists an integer $j=j_{k} \in\left(n_{k-1}, n_{k}\right)$ with $\left\|P_{j}\right\|<\frac{1}{k}$. In particular the subsequence $\left(P_{j_{k}}\right)$ is bounded. Since every limit function of $\left(F^{n}\right)$ on the Fatou component $\Omega$ is constant, it follows that for all $(z, w) \in \Omega$ we have

$$
\left\|F^{j_{k}}(z, w)-P_{j_{k}}\right\| \rightarrow 0
$$

As a consequence, we have that $\left(g_{j_{k}}\right)$ is locally uniformly bounded from above and that for all $(z, w) \in \Omega$,

$$
\limsup _{k \rightarrow \infty} g_{j_{k}}(z, w) \leq 0
$$

Lemma 7.9 We can guarantee that $g_{j_{k}} \rightarrow 0$ on $\Omega \backslash \Omega_{\left(P_{n}\right),\left(\beta_{n}\right)}$, uniformly on compact subsets. 
Proof Recall that in the construction of the wandering Fatou component the radius $\theta_{j_{k}}$ is determined before the length of the contracting detour. By making the contracting detour sufficiently long, we can guarantee that $j_{k}$ is as large as we want. For points $\left(z_{0}, w_{0}\right) \in \Omega \backslash \Omega_{\left(P_{n}\right),\left(\theta_{n}\right)}$ we have that

$$
\left\|\left(z_{j_{k}}, w_{j_{k}}\right)-P_{j_{k}}\right\| \geq \theta_{j_{k}}
$$

Thus, by making the contracting detours sufficiently long, we can guarantee that $g_{j_{k}}>-\epsilon_{k}$ on $\Omega \backslash \Omega_{\left(P_{n}\right),\left(\theta_{n}\right)}$ for any $\epsilon_{k} \searrow 0$. The conclusion follows.

Proposition 7.10 With the previous choices, the wandering Fatou component $\Omega$ equals the calibrated basin $\Omega_{\left(P_{n}\right),\left(\theta_{n}\right)}$, and is thus biholomorphically equivalent to $\mathbb{C}^{2}$.

Proof Suppose by contradiction that $\Omega \neq \Omega_{\left(P_{n}\right),\left(\theta_{n}\right)}$. Let $h$ be the upper-semicontinuous regularization of $\lim _{k \rightarrow \infty} g_{n_{k}}$. Clearly $h \equiv \log a$ on $\Omega_{\left(P_{n}\right),\left(\theta_{n}\right)}$ and $h \equiv 0$ on $\Omega \backslash \Omega_{\left(P_{n}\right),\left(\theta_{n}\right)}$. Then by [25, Prop 2.9.17] the function $h$ is plurisubharmonic, and the submean value property at any $\zeta \in \partial \Omega_{\left(P_{n}\right),\left(\theta_{n}\right)}$ gives a contradiction.

Open Access This article is distributed under the terms of the Creative Commons Attribution 4.0 International License (http://creativecommons.org/licenses/by/4.0/), which permits unrestricted use, distribution, and reproduction in any medium, provided you give appropriate credit to the original author(s) and the source, provide a link to the Creative Commons license, and indicate if changes were made.

\section{References}

1. Astorg, M., Buff, X., Dujardin, R., Peters, H., Raissy, J.: A two-dimensional polynomial mapping with a wandering Fatou component. Ann. Math. (2) 184(1), 263-313 (2016)

2. Baker, I.N.: Wandering domains in the iteration of entire functions. Proc. Lond. Math. Soc. (3) 49(3), 563-576 (1984)

3. Bedford, E.: On the automorphism group of a Stein manifold. Math. Ann. 266(2), 215-227 (1983)

4. Bedford, E., Smillie, J.: Polynomial diffeomorphisms of $\mathbb{C}^{2}$ : currents, equilibrium measure and hyperbolicity. Invent. Math. 103(1), 69-99 (1991)

5. Bedford, E., Smillie, J.: Polynomial diffeomorphisms of $\mathbb{C}^{2}$ : II. Stable manifolds and recurrence. J. Am. Math. Soc. 4(4), 657-679 (1991)

6. Bergweiler, W.: Periodic points of entire functions: proof of a conjecture of Baker. Complex Var. Theory Appl. 17(1-2), 57-72 (1991)

7. Bergweiler, W.: Iteration of meromorphic functions. Bull. Am. Math. Soc. (N.S.) 29(2), 151-188 (1993)

8. Bergweiler, W.: Quasinormal families and periodic points. In: Complex Analysis and Dynamical Systems II, Contemp. Math., vol. 382. American Mathematical Society, Providence, pp. 55-63 (2005)

9. Bergweiler, W., Zheng, J.: Some examples of Baker domains. Nonlinearity 25(4), 1033-1044 (2012)

10. Bishop, C.: Constructing entire functions by quasiconformal folding. Acta Math. 214(1), 1-60 (2015)

11. Cowen, C.C.: Iteration and the solution of functional equations for functions analytic in the unit disk. Trans. Am. Math. Soc. 265, 69-95 (1981)

12. Devaney, R.L.: Complex dynamical systems (Cincinnati, OH, 1994), pp. 181-206, Proc. Sympos. Appl. Math., vol. 49. AMS Short Course Lecture Notes, American Mathematical Society, Providence (1994)

13. Dujardin, R.: Hńon-like mappings in $\mathbb{C}^{2}$. Am. J. Math. 126(2), 439-472 (2004)

14. Eremenko, A.E.: On the iteration of entire functions, Dynamical systems and ergodic theory (Warsaw, 1986), pp. 339-345. Banach Center Publ., 23 
15. Eremenko, A.E., Lyubich, M.: Examples of entire functions with pathological dynamics. J. Lond. Math. Soc. (2) 36(3), 458-468 (1987)

16. Farkas, H.M., Kra, I.: Riemann Surfaces, 2nd edn. Graduate Texts in Mathematics, vol. 71. Springer, New York (1992)

17. Fatou, P.: Sur les équations fonctionnelles. Bull. Soc. Math. Fr. 47, 161-271 (1919)

18. Fornæss, J.E.: Short $\mathbb{C}^{k}$, Complex analysis in several variables-memorial conference of Kiyoshi Oka's centennial birthday, pp. 95-108, Adv. Stud. Pure Math., vol. 42. Math. Soc. Japan, Tokyo (2004)

19. Fornæss, J.E., Sibony, N.: Classification of recurrent domains for some holomorphic maps. Math. Ann. 301, 813-820 (1995)

20. Fornæss, J.E., Sibony, N.: Fatou and Julia sets for entire mappings in $\mathbb{C}^{k}$. Math. Ann. 311(1), 27-40 (1998)

21. Forstneric, F.: Stein manifolds and holomorphic mappings. The homotopy principle in complex analysis. Ergebnisse der Mathematik und ihrer Grenzgebiete. 3. Folge. A Series of Modern Surveys in Mathematics, vol. 56. Springer, Heidelberg (2011)

22. Friedland, S., Milnor, J.: Dynamical properties of plane polynomial automorphisms. Ergod. Theory Dyn. Syst. 9(1), 67-99 (1989)

23. Hirsch, M.W., Pugh, C.C., Shub, M.: Invariant manifolds. Bull. Am. Math. Soc. 76(5), 1015-1019 (1970)

24. Hormander, L.: An introduction to complex analysis in several variables. D. Van Nostrand, New York (1966)

25. Klimek, M.: Pluripotential theory, London Mathematical Society Monographs. New Series, 6. Oxford Science Publications. The Clarendon Press, Oxford University Press, New York, xiv+266 pp (1991)

26. Lyubich, M., Peters, H.: Classification of invariant Fatou components for dissipative Hénon maps. Geom. Funct. Anal. 24(3), 88-915 (2014)

27. Palis Jr, J., de Melo, W.: Geometric Theory of Dynamical Systems. An Introduction. Translated from the Portuguese by A. K. Manning. Springer, New York (1982)

28. Peters, H., Vivas, L.R., Wold, E.F.: Attracting basins of volume preserving automorphisms of $\mathbb{C}^{k}$. Int. J. Math. 19(7), 801-810 (2008)

29. Peters, H., Wold, E.F.: Non-autonomous basins of attraction and their boundaries. J. Geom. Anal. 15(1), 123-136 (2005)

30. Sullivan, D.: Quasiconformal homeomorphisms and dynamics. I. Solution of the Fatou-Julia problem on wandering domains. Ann. Math. 122, 401-418 (1985)

31. Weickert, B.J.: Nonwandering, nonrecurrent Fatou components in $\mathbb{P}^{2}$. Pac. J. Math. 211(2), 391-397 (2003)

32. Wiman, A.: Sur une extension d'un théorème de M. Hadamard. Arkiv för Math. Astr. och Fys. 2(14), 1-5 (1905)

33. Wold, E.F.: Fatou-Bieberbach domains. Int. J. Math. 16(10), 1119-1130 (2005) 